\title{
Thermal Hall Effects of Spins and Phonons in Kagome Antiferromagnet Cd-Kapellasite
}

\author{
Masatoshi Akazawa, ${ }^{1}$ Masaaki Shimozawa, ${ }^{1,2}$ Shunichiro Kittaka, ${ }^{1,3}$ Toshiro Sakakibara, ${ }^{1}$ Ryutaro Okuma $\odot,{ }^{1,4}$ \\ Zenji Hiroi, ${ }^{1}$ Hyun-Yong Lee $\odot,{ }^{1,5,6,7}$ Naoki Kawashima, ${ }^{1}$ Jung Hoon Han, ${ }^{8}$ and Minoru Yamashita $\oplus^{1, *}$ \\ ${ }^{1}$ The Institute for Solid State Physics, The University of Tokyo, Kashiwa, 277-8581, Japan \\ ${ }^{2}$ Graduate School of Engineering Science, Osaka University, Toyonaka, 560-8531, Japan \\ ${ }^{3}$ Department of Physics, Chuo University, Kasuga, Bunkyo-ku, Tokyo 112-8551, Japan \\ ${ }^{4}$ Okinawa Institute of Science and Technology Graduate University, Kunigami-gun, 904-0495, Japan \\ ${ }^{5}$ Department of Applied Physics, Graduate School, Korea University, Sejong 30019, Korea \\ ${ }^{6}$ Division of Display and Semiconductor Physics, Korea University, Sejong, 30019, Korea \\ ${ }^{7}$ Interdisciplinary Program in E.ICT-Culture-Sports Convergence, Korea University, Sejong 30019, Korea \\ ${ }^{8}$ Department of Physics, Sungkyunkwan University, Suwon 16419, Korea
}

(Received 11 May 2020; revised 24 September 2020; accepted 29 October 2020; published 23 December 2020)

We investigate the thermal-transport properties of the kagome antiferromagnet Cd-kapellasite (Cd-K). We find that a field-suppression effect on the longitudinal thermal conductivity $\kappa_{x x}$ sets in below approximately $25 \mathrm{~K}$. This field-suppression effect at $15 \mathrm{~T}$ becomes as large as $80 \%$ at low temperatures, suggesting a large spin contribution $\kappa_{x x}^{\mathrm{sp}}$ in $\kappa_{x x}$. We also find clear thermal Hall signals in the spin liquid phase in all $\mathrm{Cd}-\mathrm{K}$ samples. The magnitude of the thermal Hall conductivity $\kappa_{x y}$ shows a significant dependence on the sample's scattering time, as seen in the rise of the peak $\kappa_{x y}$ value in almost linear fashion with the magnitude of $\kappa_{x x}$. On the other hand, the temperature dependence of $\kappa_{x y}$ is similar in all Cd-K samples; $\kappa_{x y}$ shows a peak at almost the same temperature of the peak of the phonon thermal conductivity $\kappa_{x x}^{\mathrm{ph}}$ which is estimated by $\kappa_{x x}$ at $15 \mathrm{~T}$. These results indicate the presence of a dominant phonon thermal Hall $\kappa_{x y}^{\mathrm{ph}}$ at $15 \mathrm{~T}$. In addition to $\kappa_{x y}^{\mathrm{ph}}$, we find that the field dependence of $\kappa_{x y}$ at low fields turns out to be nonlinear at low temperatures, concomitantly with the appearance of the field suppression of $\kappa_{x x}$, indicating the presence of a spin thermal Hall $\kappa_{x y}^{\mathrm{sp}}$ at low fields. Remarkably, by assembling the $\kappa_{x x}$ dependence of $\kappa_{x y}^{\mathrm{sp}}$ data of other kagome antiferromagnets, we find that, whereas $\kappa_{x y}^{\mathrm{sp}}$ stays a constant in the low- $\kappa_{x x}$ region, $\kappa_{x y}^{\mathrm{sp}}$ starts to increase as $\kappa_{x x}$ does in the high- $\kappa_{x x}$ region. This $\kappa_{x x}$ dependence of $\kappa_{x y}^{\mathrm{sp}}$ indicates the presence of both intrinsic and extrinsic mechanisms in the spin thermal Hall effect in kagome antiferromagnets. Furthermore, both $\kappa_{x y}^{\mathrm{ph}}$ and $\kappa_{x y}^{\mathrm{sp}}$ disappear in the antiferromagnetic ordered phase at low fields, showing that phonons alone do not exhibit the thermal Hall effect. A high field above approximately $7 \mathrm{~T}$ induces $\kappa_{x y}^{\mathrm{ph}}$, concomitantly with a field-induced increase of $\kappa_{x x}$ and the specific heat, suggesting a coupling of the phonons to the field-induced spin excitations as the origin of $\kappa_{x y}^{\mathrm{ph}}$.

DOI: $10.1103 /$ PhysRevX.10.041059

\section{INTRODUCTION}

The magnetic ground state of a two-dimensional (2D) kagome structure has been attracting tremendous attention, because the strong frustration effect caused by the cornersharing network of the triangles has been expected to suppress the magnetic order even at absolute zero temperature. Instead of a long-range ordered state, the emergence

*my@issp.u-tokyo.ac.jp

Published by the American Physical Society under the terms of the Creative Commons Attribution 4.0 International license. Further distribution of this work must maintain attribution to the author(s) and the published article's title, journal citation, and DOI.
Subject Areas: Condensed Matter Physics, Magnetism, Strongly Correlated Materials of a quantum disordered state of spins, termed as a quantum spin liquid (QSL), is shown in the kagome Heisenberg antiferromagnet (KHA) by various numerical calculations [1-8]. A lot of QSLs are theoretically suggested as the ground state of the KHA such as $\mathbb{Z}_{2}$ spin liquids $[2,9,10]$, topological spin liquids [3], Dirac spin liquids [4,6-8], and chiral spin liquids [11]. These different QSLs are characterized by different elementary excitations. It is, thus, an experimental challenge to pin down the QSL realized in the KHA by clarifying the elementary excitation.

Thermal-transport measurement is a powerful probe to study the elementary excitations in QSLs, because it has the advantage of detecting only the itinerant excitations. Therefore, one can avoid effects of localized excitations caused by impurities which are often inevitable in 
candidate materials [12]. Moreover, further details of the elementary excitation can be studied by investigating the thermal Hall effect. It is shown that the thermal Hall effect in an insulator is given by the Berry curvature of the elementary excitation as

$$
\kappa_{x y}=\frac{k_{B} T}{\hbar V} \sum_{k} \sum_{n} c_{2}\left[g\left(\epsilon_{n k}\right)\right] \Omega_{n k},
$$

where $c_{2}\left[g\left(\epsilon_{n k}\right)\right]$ is a distribution function given by the elementary excitations of energy $\epsilon_{n k}$ and $\Omega_{n k}$ is the Berry curvature of the elementary excitations [13,14]. Therefore, from $\kappa_{x y}$ measurements, one can study the statics of the elementary excitations (fermions or bosons) as well as the Berry curvature of the corresponding energy bands [15-21].

The thermal Hall effect of spins $\left(\kappa_{x y}^{\mathrm{sp}}\right)$ is observed in ferromagnetic insulators, which is well understood as a magnon thermal Hall effect [22,23]. The spin thermal Hall effect is also reported in paramagnetic states of kagome [24-27], spin ice [28], and Kitaev compounds [29-31]. In these frustrated magnets, the paramagnetic phase extends well below the temperatures determined by the interaction energy $J$, realizing a spin liquid phase in a wide temperature range $T_{N} \leq T \ll J / k_{B}$. For $\kappa_{x y}$ observed in the spin liquid phase of kagome antiferromagnets volborthite and $\mathrm{Ca}$ kapellasite $(\mathrm{Ca}-\mathrm{K})$, it is shown that the Schwinger-boson mean-field theory (SBMFT) [32] can well reproduce both the temperature dependence and the magnitude of $\kappa_{x y}$ by tuning the two fitting parameters of the spin interaction energy $J$ and the Dzyaloshinskii-Moriya (DM) interaction $D$ (Ref. [26]). Remarkably, the fitting results of $J$ and $D$, obtained by the SBMFT fitting to $\kappa_{x y}$ of both kagome compounds, are close to the values estimated by the temperature dependence of the magnetic susceptibility and that by the deviation of the $g$ factor, respectively. This excellent agreement suggests that the elementary spin excitations in the KHA can be well described by the bosonic spinons of SBMFT.

In addition to the spin thermal Hall effects, the thermal Hall effects of phonons $\left(\kappa_{x y}^{\mathrm{ph}}\right)$ are reported in various compounds [33-37]. The origin of the phonon thermal Hall is also extensively studied theoretically [38-46]. However, the understanding of the phonon thermal Hall effect is left out in the consideration of the spin thermal Hall effect, because the nature of the coupling between phonons and spin fluctuations remains unclear.

In this article, we report our thermal-transport measurements of a new kagome antiferromagnet Cd-kapellasite (Cd-K). Previous studies [47,48] show that the spin Hamiltonian of $\mathrm{Cd}-\mathrm{K}$ is well approximated to a KHA with the spin interaction energy of $J / k_{B} \sim 45 \mathrm{~K}$. The frustration effect of the kagome structure suppresses the ordering temperature $\left(T_{N} \sim 4 \mathrm{~K}\right)$ well below $J / k_{B}$, realizing a spin liquid phase in a wide temperature range. We find a large spin contribution in $\kappa_{x x}$ which can be strongly suppressed by applying a magnetic field. This field-suppression effect on $\kappa_{x x}^{\mathrm{sp}}$ allows us to identify both $\kappa_{x y}^{\mathrm{sp}}$ and $\kappa_{x y}^{\mathrm{ph}}$ in Cd-K. Most remarkably, we find the $\kappa_{x x}$ dependence of $\kappa_{x y}^{\mathrm{sp}}$ indicates the presence of both intrinsic and extrinsic mechanisms depending on the strength of the impurity scatterings for the spin thermal Hall effect. Furthermore, we find that both $\kappa_{x y}^{\mathrm{sp}}$ and $\kappa_{x y}^{\mathrm{ph}}$ disappear in the AFM phase at low fields. Applying a high field in the AFM phase induces $\kappa_{x y}^{\mathrm{ph}}$, concomitantly with the appearance of additional excitations probed by the specific heat and $\kappa_{x x}$. We conclude that Cd-K is a prominent frustrated magnet in which the spin liquid state shows thermal Hall effects of both spins and phonons. The dual nature of the thermal conductivities prompts us to speculate that a spin-phonon coupling gives rise to both $\kappa_{x y}^{\mathrm{sp}}$ and $\kappa_{x y}^{\mathrm{ph}}$ in this compound.

\section{MATERIALS AND METHODS}

Cd-kapellasite $\mathrm{CdCu}_{3}(\mathrm{OH})_{6}\left(\mathrm{NO}_{3}\right)_{2} \cdot \mathrm{H}_{2} \mathrm{O}$ is a trigonal compound with space group $P \overline{3} m 1$ and lattice constants $a=6.5449 \AA$ and $c=7.0328 \AA$ [47], in which the magnetic $\mathrm{Cu}^{2+}$ ions form an undistorted kagome lattice [Fig. 1(a)]. Cd-kapellasite is isostructural to Zn-kapellasite (a)

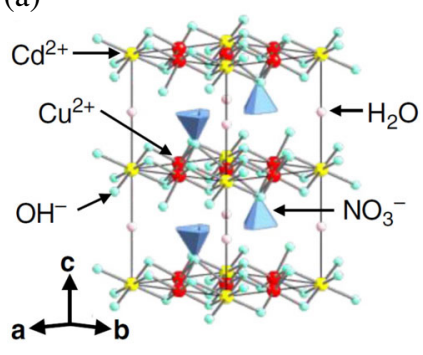

(c)

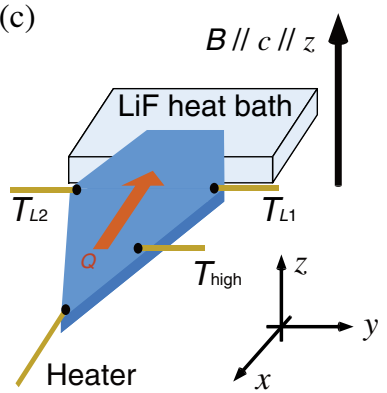

(b)

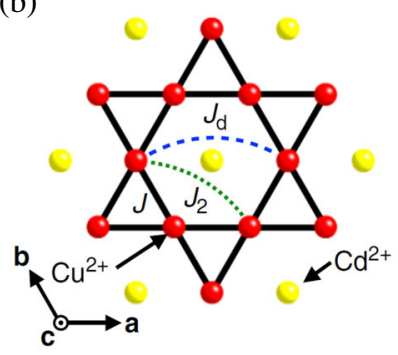

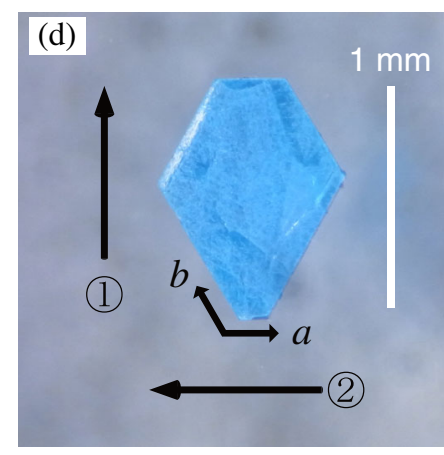

FIG. 1. (a) Crystal structure and (b) top view of a kagome layer of $\mathrm{Cd}-\mathrm{K}$ [48]. The magnetic interactions between nearestneighbor, next-nearest-neighbor, and diagonal $\mathrm{Cu}^{2+}$ spins are denoted by $J$ (solid black line), $J_{2}$ (dotted line), and $J_{d}$ (dashed line), respectively. (c) Schematic illustration of $\kappa_{x x}$ and $\kappa_{x y}$ measurements. A heater and three thermometers $\left(T_{\text {high }}, T_{L 1}\right.$, and $\left.T_{L 2}\right)$ are attached to the sample fixed on the LiF heat bath. A heat current $Q$ is applied within the kagome layer, and a magnetic field $B$ is applied along the $c$ axis. (d) A typical crystal of Cd-K. The direction of the heat current for samples 1, 2, and 3-1 (3-2) is shown by arrow 1 (2). 
$\mathrm{ZnCu}_{3}(\mathrm{OH})_{6} \mathrm{Cl}_{2}$, which is a polymorph of herbertsmithite [49]. In herbertsmithite, the $\mathrm{Zn}$ ions located between the kagome layer and the site mixings between the $\mathrm{Zn}$ and $\mathrm{Cu}$ ions [12] allow an interlayer coupling between the kagome layers. In contrast, in $\mathrm{Cd}-\mathrm{K}$, the nonmagnetic $\mathrm{Cd}$ ions are located at the center of the hexagon of the kagome lattice and there are no site mixings in $\mathrm{Cd}-\mathrm{K}$ because of the larger ionic radii of $\mathrm{Cd}^{2+}(0.95 \AA)$ than that of $\mathrm{Cu}^{2+}(0.73 \AA)$ [50], realizing a more ideal $\mathrm{KHA}$ in $\mathrm{Cd}-\mathrm{K}$.

Three magnetic interactions in $\mathrm{Cd}-\mathrm{K}$ are suggested by the fitting of the magnetic susceptibility as $J / k_{B}=45.44 \mathrm{~K}$, $J_{2} / J=-0.1$, and $J_{d} / J=0.18$, where $J$ is the nearestneighbor interaction, $J_{2}$ the next-nearest-neighbor interaction, and $J_{d}$ the diagonal interaction via the nonmagnetic $\mathrm{Cd}$ ion [see Fig. 1(b)]. The development of a short-range antiferromagnetic correlation is shown by the decrease of the magnetic susceptibility below $30 \mathrm{~K}$ [47]. The $g$ factors are estimated as $g_{a}=2.28$ and $g_{c}=2.37$ [48]. The lack of inversion symmetry allows both the in-plane and out-ofplane DM interactions, which is suggested to cause a negative vector chiral order below the Néel temperature of $T_{N} \sim 4 \mathrm{~K}$ [47].

The thermal-transport measurements are performed by the steady-state method as described in Refs. [25-27]. One heater and three thermometers are attached to the sample, and then the temperature differences $\Delta T_{x}\left(\Delta T_{x}=T_{\text {high }}-T_{L 1}\right)$ and $\Delta T_{y}\left(\Delta T_{y}=T_{L 1}-T_{L 2}\right)$ are measured by applying the heat current $Q$ in the kagome plane [Fig. 1(c)]. The longitudinal thermal conductivity $\kappa_{x x}$ and the thermal Hall conductivity $\kappa_{x y}$ are derived by

$$
\left(\begin{array}{c}
Q / w t \\
0
\end{array}\right)=\left(\begin{array}{cc}
\kappa_{x x} & \kappa_{x y} \\
-\kappa_{x y} & \kappa_{x x}
\end{array}\right)\left(\begin{array}{c}
\Delta T_{x} / L \\
\Delta T_{y}^{\text {asym }} / w^{\prime}
\end{array}\right)
$$

where $t$ is the thickness of the sample, $L$ is the length between $T_{\text {high }}$ and $T_{L 1}, w$ is the averaged sample width between $T_{L 1}$ and $T_{L 2}, w^{\prime}$ is the length between $T_{L 1}$ and $T_{L 2}$, and $\Delta T_{y}^{\text {asym }}$ is the antisymmetrized $\Delta T_{y}$ with respect to the field direction as $\Delta T_{y}^{\text {asym }}(B)=\left[\Delta T_{y}(+B)-\Delta T_{y}(-B)\right] / 2$.

We measure $\kappa_{x x}$ and $\kappa_{x y}$ of three Cd-K samples (samples 1,2 , and 3 ) by using a variable temperature insert (VTI) (2-60 K, 0-15 T). Measurements of sample 2 are also done in a dilution refrigerator (DR) $(0.1-4 \mathrm{~K}, 0-14 \mathrm{~T})$. The magnetic field is applied along the $c$ axis of the sample. A typical sample is shown in Fig. 1(d). Because of the nonrectangular shape of the sample, there is an ambiguity up to about $40 \%$ in estimating the absolute value of $\kappa_{x x}$ and $\kappa_{x y}$ (see Supplemental Material [51] for more details). A heat current $Q$ is applied along direction $1[\perp a$ axis; see Fig. 1(d)] in samples 1 and 2 and the first run of sample 3 (denoted as sample 3-1). In the second run of sample 3 (sample 3-2), the direction of $Q$ is changed to direction 2 [ $\| a$ axis; see Fig. 1(d)]. For each $\kappa_{x x}$ and $\kappa_{x y}$ measurement, we confirm the linear $Q$ dependence of both $\Delta T_{x}$ and
$\Delta T_{y}^{\text {asym }}$ (see Fig. S1 in Supplemental Material [51]). We also check that the temperature stability during the measurements is good enough to resolve $\Delta T_{y}^{\text {asym }}$ (Fig. S2 in Supplemental Material [51]).

The specific heat measurements are performed for two sets of multiple single crystals by a thermal relaxation method by using a physical property measurement system (PPMS, Quantum Design) and a DR. The PPMS measurements $(2-10 \mathrm{~K}, 0-10 \mathrm{~T})$ are performed for the same set of single crystals used in Ref. [47]. The DR measurements $(0.1-2 \mathrm{~K}, 0-14 \mathrm{~T})$ are performed for another set of single crystals. The magnetic field is applied along the $c$ axis of the samples in all the measurements.

\section{RESULTS}

\section{A. Longitudinal thermal conductivity}

Figure 2(a) shows the temperature dependence of $\kappa_{x x}$ of all Cd-K samples at zero magnetic field. For reference, $\kappa_{x x}$ of Ca-K [26] is also shown. As shown in Fig. 2(a), $\kappa_{x x}$ of all $\mathrm{Cd}-\mathrm{K}$ samples is about one order of magnitude larger than that of $\mathrm{Ca}-\mathrm{K}$. Although the magnitudes of $\kappa_{x x}$ in different $\mathrm{Cd}-\mathrm{K}$ samples are different by a factor of approximately 2 , $\kappa_{x x}$ of all Cd-K samples show a similar temperature dependence. The temperature dependence of $\kappa_{x x}$ shows a shoulderlike enhancement around $15 \mathrm{~K}$, which is followed by a hump near $T_{N}$ and a rapid decrease for $T<T_{N}$.

The temperature dependence of $\kappa_{x x}$ at different magnetic fields is shown in Figs. 2(b)-2(f). In all Cd-K samples, a decrease of $\kappa_{x x}$ by applying the magnetic field is observed below approximately $25 \mathrm{~K}$. This field-suppression effect is larger for a sample with a large $\kappa_{x x}$. In sample 2, additional lower-temperature measurements are performed by using a DR [open circles in Figs. 2(c) and 2(d)]. As shown in Fig. 2(d), a large field-suppression effect is observed at approximately $1 \mathrm{~K}$.

Figure 3 shows the magnetic field dependence of $\kappa_{x x}$ of sample 2 . The vertical axis is normalized by the zero-field value as $\left[\kappa_{x x}(B)-\kappa_{x x}(0)\right] / \kappa_{x x}(0)$. The field dependence of $\kappa_{x x}$ of other samples is essentially the same. Above $40 \mathrm{~K}, \kappa_{x x}$ increases linearly by applying the magnetic field [Fig. 3(a)]. On the other hand, below approximately $25 \mathrm{~K}$, the suppression of $\kappa_{x x}$ by the magnetic field is observed [Fig. 3(b)]. The field-suppression effect becomes larger at lower temperatures and reaches the maximum reduction of approximately $80 \%$ by $15 \mathrm{~T}$ at $1 \mathrm{~K}$. Below $0.3 \mathrm{~K}$, a new peak is observed in the field dependence of $\kappa_{x x}$ at 6-7 T [Fig. 3(c)].

\section{B. Thermal Hall conductivity}

Figure 4(a) shows the magnetic field dependence of $\Delta T_{y} / Q$ of sample 2 in the spin liquid phase. As shown in Fig. 4(a), the field dependence of $\Delta T_{y} / Q$ is dominated by the symmetric longitudinal component caused by the misalignment effect. To extract the asymmetric thermal Hall effect, the field dependence of $\Delta T_{y} / Q$ is 

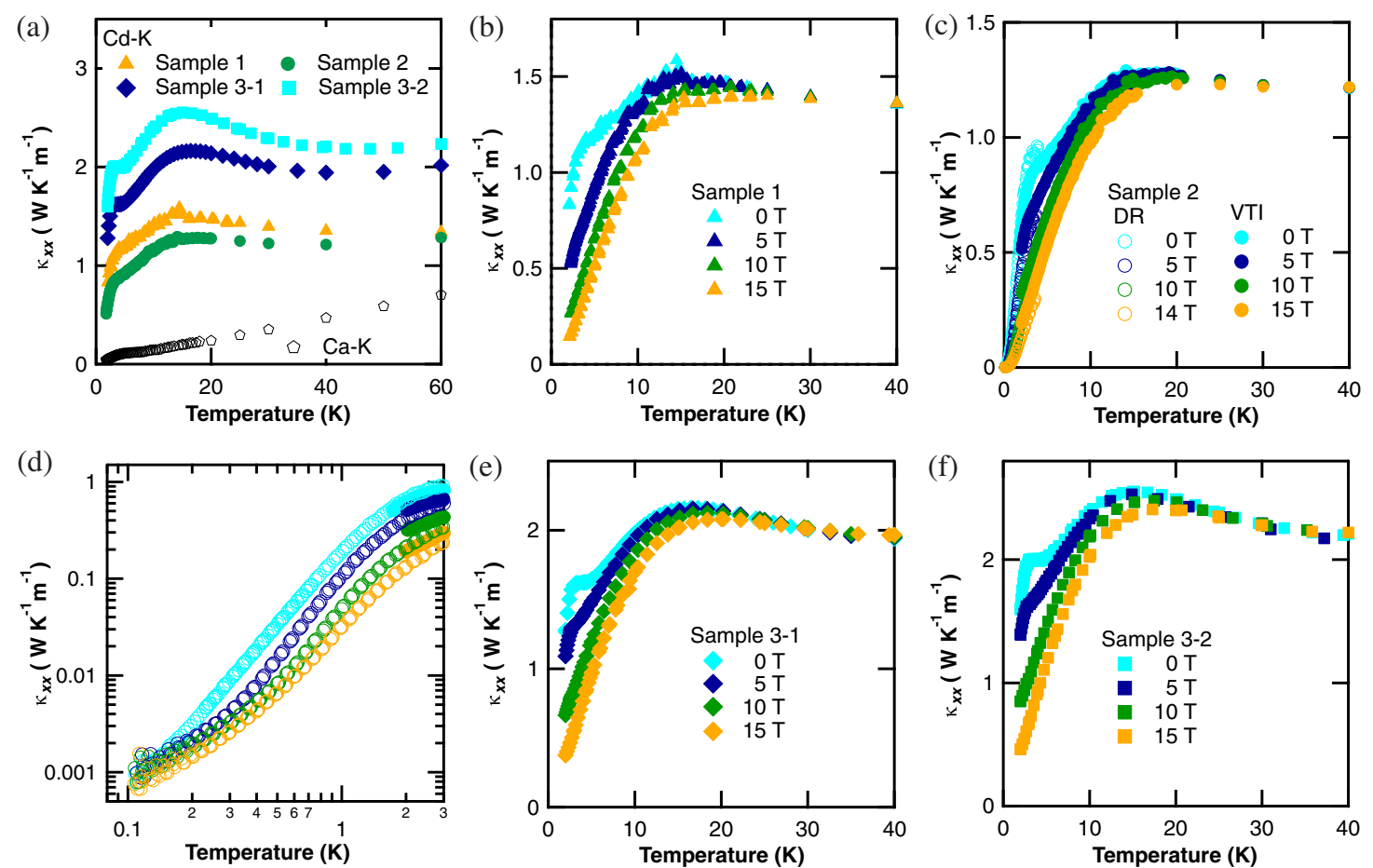

FIG. 2. (a) Temperature dependence of the longitudinal thermal conductivity $\left(\kappa_{x x}\right)$ of all samples of Cd-kapellasite (Cd-K) and that of Ca-kapellasite (Ca-K) at $0 \mathrm{~T}$. The longitudinal thermal conductivity of Ca-K is taken from Ref. [26]. (b)-(f) The data of each sample under magnetic fields. In sample $2, \kappa_{x x}$ is measured down to $0.1 \mathrm{~K}$. An enlarged view of the low-temperature region $(0.1-3 \mathrm{~K})$ of $(\mathrm{c})$ is shown in (d). The filled and open circles in (c) and (d) show $\kappa_{x x}$ measured by a VTI $(2 \mathrm{~K}<T)$ and a DR $(0.1<T<4 \mathrm{~K})$, respectively. The slight difference between the two data might be caused by a thermal cycle effect and/or different setups between the VTI and the DR measurements.

antisymmetrized with respect to the field direction as $\Delta T_{y}^{\text {asym }}(B)=\left[\Delta T_{y}(+B)-\Delta T_{y}(-B)\right] / 2$. The field dependence of $\Delta T_{y}^{\text {asym }}(B)$ of sample 2 is shown in Fig. 4(b). As shown in Fig. 4(b), $\Delta T_{y}^{\text {asym }} / Q$ shows a linear magnetic field dependence at high temperatures.

The field dependence of $\kappa_{x y}$ is determined by $\Delta T_{y}^{\text {asym }}$ in accordance with Eq. (2) and is plotted in Fig. 5. As shown in Fig. 5, the linear field dependence of $\kappa_{x y}$ (solid lines in Fig. 5) is observed at $20 \mathrm{~K}$, which becomes nonlinear at lower temperatures.
This thermal Hall signal disappears in the AFM phase at low fields. Figure 4(c) shows the field dependence of $\Delta T_{y}^{\text {asym }}$ of sample 2 measured in a dilution refrigerator. As shown in Fig. 4(c), the thermal Hall effect is absent at low fields, which is followed by an increase above approximately $7 \mathrm{~T}$. A similar nonlinear field dependence is confirmed in all Cd-K samples done at the lowest temperature of the VTI measurement $(2 \mathrm{~K})$ as shown in Fig. 6.

Figure 7(a) shows the temperature dependence of $\kappa_{x y} / T B$ of all Cd-K samples at 15 (14) $\mathrm{T}$ for the VTI
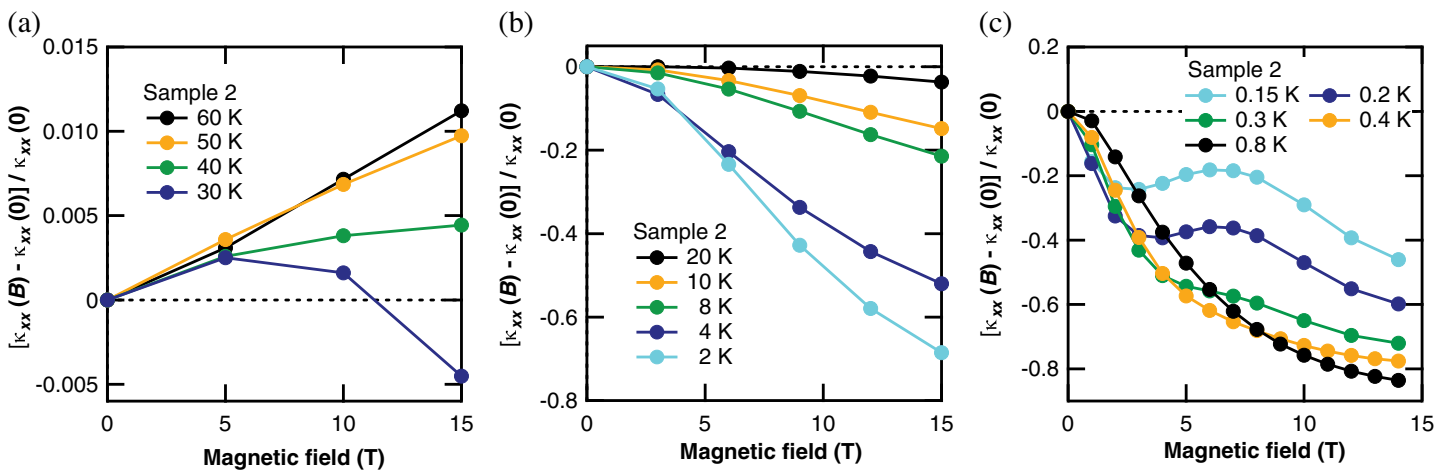

FIG. 3. Magnetic field dependence of the longitudinal thermal conductivity normalized by the zero-field value $\left\{\left[\kappa_{x x}(B)-\right.\right.$ $\left.\left.\kappa_{x x}(0)\right] / \kappa_{x x}(0)\right\}$ of sample 2 above $30 \mathrm{~K}(\mathrm{a})$, for $2-20 \mathrm{~K}(\mathrm{~b})$, and below $2 \mathrm{~K}(\mathrm{c})$. 

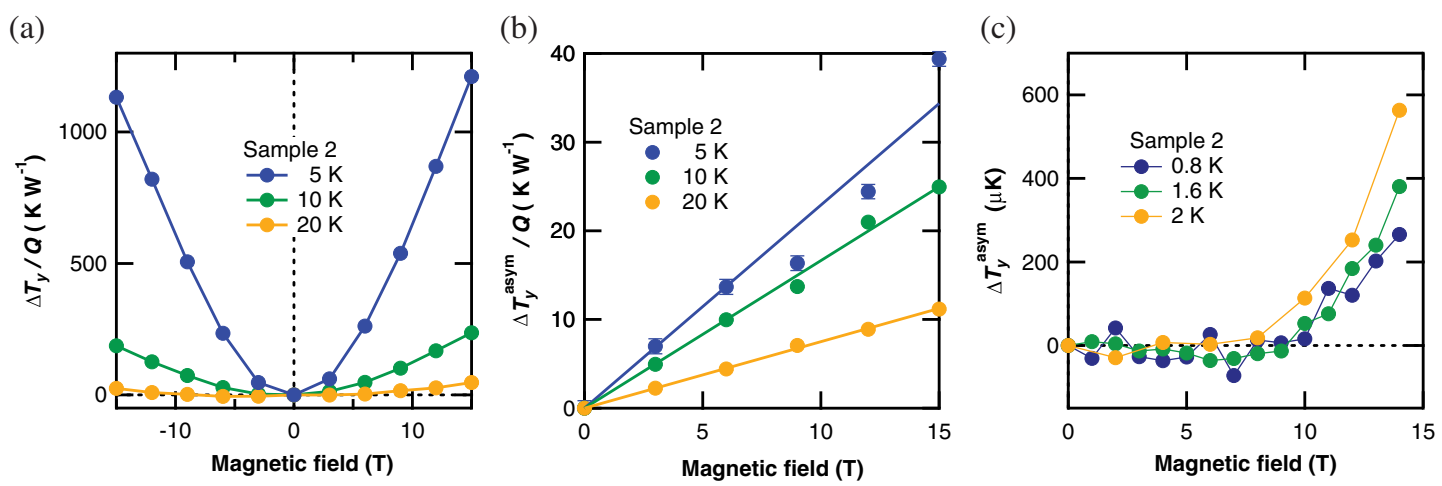

FIG. 4. (a) Magnetic field dependence of the transverse temperature difference divided by the heat current $\left(\Delta T_{y} / Q\right)$. The zero-field value of $\Delta T_{y} / Q$, which is caused by the misalignment effect, is subtracted for clarity. (b) Magnetic field dependence of the asymmetrized $\Delta T_{y} / Q$ of (a) with respect to the field direction. See the text for details. The solid lines represent a linear fitting to $\Delta T_{y}^{\mathrm{asym}} / Q$ for each temperature. (c) Magnetic field dependence of $\Delta T_{y}^{\mathrm{asym}}$ below $2 \mathrm{~K}$. Error bars represent the standard error of the data, which are smaller than the symbol size except for the $5 \mathrm{~K}$ data in (b).
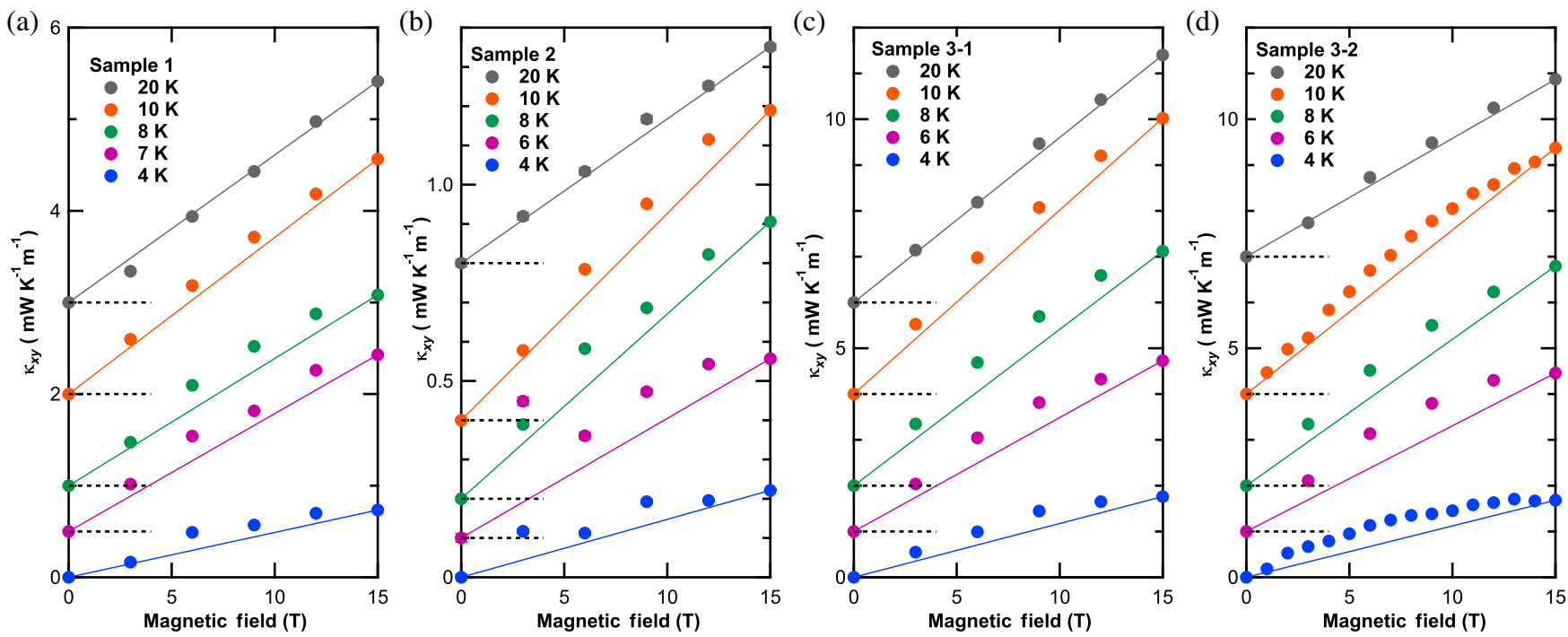

FIG. 5. (a-d) The field dependence of $\kappa_{x y}$ of all Cd-K samples for 4-20 K. The data above $4 \mathrm{~K}$ are shifted for clarity. The offsets for the shifted data are indicated by the dashed lines. The solid lines are drawn to show a deviation from the linear increase of $\kappa_{x y}$ to that at $15 \mathrm{~T}$. Error bars estimated by the standard error are smaller than the symbol size for all the measurements.

(DR) measurements. As shown in Fig. 7(a), $\kappa_{x y} / T B$ of all Cd-K samples shows a similar temperature dependence with a peak around $8 \mathrm{~K}$. In sample $2, \kappa_{x y} / T B$ obtained in the ordered phase at $14 \mathrm{~T}$ done by the $\mathrm{DR}$ measurements is also shown by open symbols, which seems to be smoothly connected to the VTI data shown by filled symbols [see the inset in Fig. 7(b)]. This temperature dependence is also similar to that of $\mathrm{Ca}-\mathrm{K}$ [26] and volborthite [25] [Fig. 7(b)]. On the other hand, as shown in Fig. 7(b), the peak temperature of $\kappa_{x y} / T B$ is clearly shifted to a lower temperature in $\mathrm{Cd}-\mathrm{K}$.

\section{Specific heat}

Figure 8(a) shows the temperature dependence of the specific heat divided by the temperature $(C / T)$ measured in
PPMS. As shown in Fig. 8(a), although the peak in $C / T$ by the AFM transition becomes broader at higher fields, the peak temperature does not depend on the magnetic field up to $5 \mathrm{~T}$, which is followed by a slight increase of $T_{N}$.

Because of the absence of a nonmagnetic compound isostructural to $\mathrm{Cd}-\mathrm{K}$, we estimate the phonon specific heat $C_{\mathrm{ph}} / T$ by fitting the data at high temperatures (see Supplemental Material [51] for details). As shown by the dashed line in Fig. 8(a), $C_{\mathrm{ph}} / T$ is considerably smaller than $C / T$ below $10 \mathrm{~K}$, showing that the specific heat is dominated by the magnetic contribution.

Figure 8(b) shows the temperature dependence of $C / T$ of another set of multiple single crystals measured in DR. The zero-field data show good agreement with the previous data 


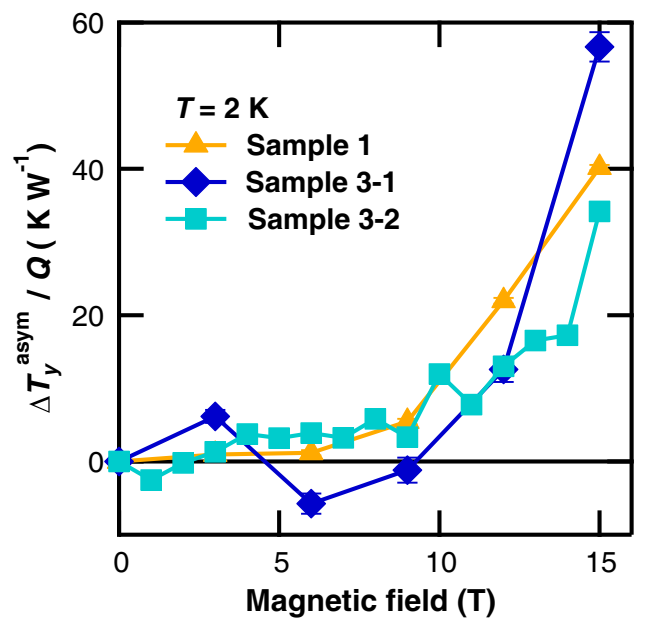

FIG. 6. Magnetic field dependence of the asymmetrized transverse temperature difference divided by the heat current $\left(\Delta T_{y}^{\text {asym }} / Q\right)$ at $2 \mathrm{~K}$. Error bars represent the standard error of the measurements.

[47] shown as open circles in Fig. 8(b). Below 0.5 K, $C / T$ under magnetic fields increases rapidly as lowering temperature owing to the nuclear Schottky anomaly $\left(C_{\mathrm{ncl}}\right)$. The magnetic field dependence of the specific heat at $0.5 \mathrm{~K}$ is shown in Fig. 8(c). After the specific heat is decreased by applying the magnetic field, the specific heat is increased by applying the magnetic field above $7 \mathrm{~T}$. We note that this field increase of $C$ above $7 \mathrm{~T}$ is much larger than that expected by $C_{\mathrm{ncl}}$ [dotted line in Fig. 8(c)], which is estimated as approximately $2 \mathrm{~mJ} \mathrm{~K}^{-1} \mathrm{~mol}^{-1}$ at $0.5 \mathrm{~K}$ and $10 \mathrm{~T}$ from the fit of $C_{\mathrm{ncl}} \propto H^{2} / T^{2}$ for the data shown in Fig. 8(b).

\section{D. $B$ - $T$ phase diagram of $\mathrm{Cd}-\mathrm{K}$}

From the specific heat measurements at different magnetic fields [Fig. 8(a)] and the field dependence of $\Delta T_{y}^{\text {asym }}$ [Fig. 4(c)], we determine the $B-T$ phase diagram of Cd-K (Fig. 9). As shown in Fig. 9, $T_{N}$ determined by the peak temperature of $C / T$ slightly increases above $5 \mathrm{~T}$. A similar increase of $T_{N}$ is observed in kapellasite [52] and Ca-K [53]. The threshold field of $\Delta T_{y}^{\text {asym }}$ (gray circles) seems to gradually decrease to zero as $T$ increases to $T_{N}$.

\section{DISCUSSION}

\section{A. Longitudinal thermal conductivity}

First, we discuss the sample dependence of $\kappa_{x x}$ [Fig. 2(a)] in terms of the sample quality. The longitudinal thermal conductivity of an insulator is given by the sum of the contribution of the phonons $\kappa_{x x}^{\mathrm{ph}}$ and that of the spins $\kappa_{x x}^{\mathrm{sp}}$ [54]. Considering $J / k_{B} \sim 45 \mathrm{~K}$, it can be expected that $\kappa_{x x}$ above $45 \mathrm{~K}$ is almost given by $\kappa_{x x}^{\mathrm{ph}}$, which is consistent with the field dependence of $\kappa_{x x}$. It is known that $\kappa_{x x}^{\mathrm{ph}}$ increases in the magnetic field, because the spin-phonon scatterings are reduced under the magnetic field by suppressing spin fluctuations [55]. In fact, as shown in Fig. 3(a), the increase of $\kappa_{x x}$ by applying the magnetic field is observed above $40 \mathrm{~K}$, showing a dominant $\kappa_{x x}^{\mathrm{ph}}$ in $\kappa_{x x}$ at high temperatures.

The phonon thermal conductivity $\kappa_{x x}^{\mathrm{ph}}$ is given by a product of the specific heat $C_{\mathrm{ph}}$, the mean free path $\ell_{\mathrm{ph}}$, and the velocity $v_{\mathrm{ph}}$ of phonons, as $\kappa_{x x}^{\mathrm{ph}}=(1 / 3) C_{\mathrm{ph}} l_{\mathrm{ph}} v_{\mathrm{ph}}$. Since $C_{\mathrm{ph}}$ and $v_{\mathrm{ph}}$ are common in all Cd-K samples, the difference in the magnitude of $\kappa_{x x}$ shown in Fig. 2(a) reflects the difference in $\ell_{\mathrm{ph}}$ of each sample. Therefore, a sample with a larger $\kappa_{x x}$ is a better crystal with fewer impurities. Also, the

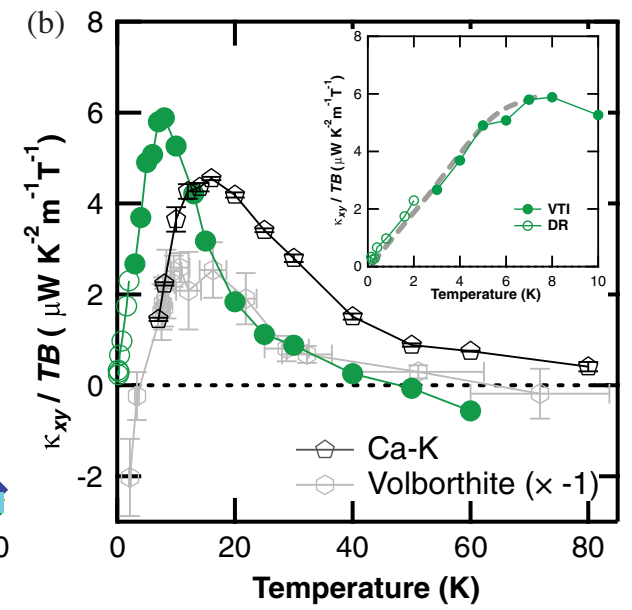

FIG. 7. Temperature dependence of $\kappa_{x y} / T B$. (a) Comparison of $\kappa_{x y} / T B$ of three Cd-K samples. The filled (open) symbols represent $\kappa_{x y} / T B$ at $15 \mathrm{~T}$ in the VTI measurements (14 T in the DR measurements). (b) Comparison of $\kappa_{x y} / T B$ of Cd-K sample 2 (green circles), $\mathrm{Ca}-\mathrm{K}[26]$ (open gray pentagon), and volborthite [25] (open gray hexagon). For clarity, $\kappa_{x y} / T B$ of volborthite is multiplied by -1 . The inset shows an enlarged view of the low-temperature data of $\kappa_{x y} / T B$ of sample 2 . The dashed line is a guide to the eye. 

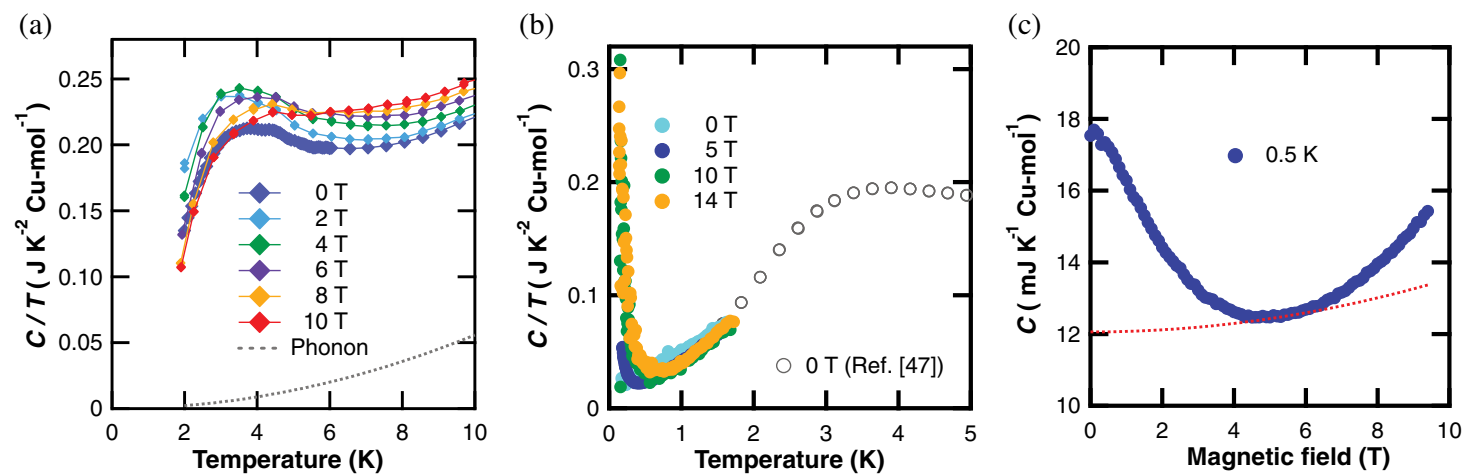

FIG. 8. (a) Temperature dependence of the specific heat divided by the temperature $(C / T)$ measured in PPMS. The measurements are done for the same set of multiple single crystals used in Ref. [47]. The peak around $4 \mathrm{~K}$ corresponds to the AFM transition. The dashed line shows the phonon contribution $C_{\mathrm{ph}} / T$ estimated by a fitting for the data at high temperatures (see Supplemental Material [51] for details). (b) Temperature dependence of $C / T$ of another set of multiple single crystals measured in DR. The zero-field data from the previous report [47] are also shown by open circles. (b) Magnetic field dependence of $C$ at $0.5 \mathrm{~K}$. The red dotted line shows an estimation of the magnetic field dependence of the nuclear Schottky specific heat $\left(C_{\mathrm{ncl}}\right)$ at $0.5 \mathrm{~K}$. The data of $C_{\mathrm{ncl}}$ are shifted to compare the amount of the field increase.

larger $\kappa_{x x}$ of Cd-K than that of $\mathrm{Ca}-\mathrm{K}$ shows that $\ell_{\mathrm{ph}}$ of $\mathrm{Cd}-\mathrm{K}$ is much longer than that of Ca-K, because $C_{\mathrm{ph}}$ and $v_{\mathrm{ph}}$ of $\mathrm{Cd}-\mathrm{K}$ are similar to those of the isostructural $\mathrm{Ca}-\mathrm{K}$. This longer $\ell_{\mathrm{ph}}$ of $\mathrm{Cd}-\mathrm{K}$ than that of $\mathrm{Ca}-\mathrm{K}[26]$ indicates that $\mathrm{Cd}-\mathrm{K}$ has a more ideal kagome structure without the randomness of ions or the lattice defects found in $\mathrm{Ca}-\mathrm{K}$ [56]. We note that the difference of $\kappa_{x x}$ of sample 3-1 and that of sample 3-2 might be caused by the ambiguity in estimating the sample size (up to 10\%) owing to the irregular shape of the sample [see Fig. 1(d)].

Next, we discuss the field-suppression effect on $\kappa_{x x}$ observed below approximately $25 \mathrm{~K}$ [Fig. 3(b)]. One of the field-suppression mechanisms of $\kappa_{x x}^{\mathrm{ph}}$, which normally increases in the magnetic field, is a resonance scattering of phonons being absorbed by impurity free spins [55].

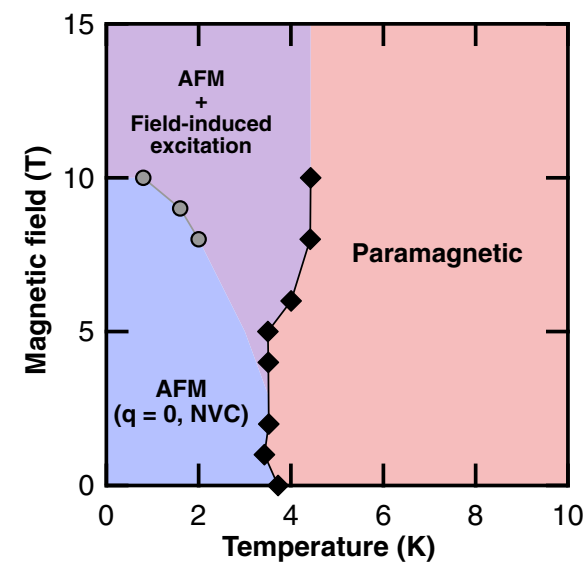

FIG. 9. $\quad B-T$ phase diagram of Cd-K. The boundary of the AFM phase is determined by the peak temperature of $C / T$ (black diamonds) shown in Fig. 8. The threshold fields where the onset of the finite $\Delta T_{y}^{\mathrm{asym}}$ is observed in the field dependence [Fig. 4(c)] are shown by gray circles.
This resonant scattering is most effective when the spin Zeeman gap $\left(g \mu_{B} H\right)$ coincides with the phonon peak (approximately $4 k_{B} T$ ) given by the Debye distribution, where $\mu_{B}$ is the Bohr magneton. Therefore, this resonance scattering produces a suppression peak of $\kappa_{x x}$ at $5.4 \mathrm{~T}$ for $2 \mathrm{~K}$ as observed in volborthite [25]. However, as shown in Fig. 3(b), $\kappa_{x x}$ at $2 \mathrm{~K}$ decreases monotonically with increasing magnetic field up to $15 \mathrm{~T}$ without the expected suppression peak. Therefore, the field-suppression effect of $\kappa_{x x}$ cannot be explained by the resonance scattering effect on $\kappa_{x x}^{\mathrm{ph}}$. Therefore, the field-suppression effect is caused by the decrease of $\kappa_{x x}^{\mathrm{sp}}$ under magnetic fields. A similar fieldsuppression effect on $\kappa_{x x}^{\mathrm{sp}}$ is also observed in the spin liquid state of the one-dimensional (1D) spin-chain compound [57], volborthite [25,27], and Ca-K [26]. In volborthite, a field-suppression effect up to approximately $30 \%$ at $15 \mathrm{~T}$ is observed [27] together with the resonance scattering effect [25]. Compared to the field-suppression effects in volborthite and $\mathrm{Ca}-\mathrm{K}$, the field suppression of $\kappa_{x x}$ in $\mathrm{Cd}-\mathrm{K}$ is much larger (approximately $80 \%$ at approximately $1 \mathrm{~K}$ ), showing a dominant contribution of $\kappa_{x x}^{\mathrm{sp}}$ in $\kappa_{x x}$ at low temperatures.

The thermal conduction of spin is also given by $\kappa_{x x}^{\mathrm{sp}}=C_{\mathrm{sp}} v_{\mathrm{sp}} \ell_{\mathrm{sp}} / 3$, where $C_{\mathrm{sp}}, v_{\mathrm{sp}}$, and $\ell_{\mathrm{sp}}$ are the specific heat, the velocity, and the mean free path of the spin excitations, respectively. As shown in Fig. 8(a), the specific heat does not show a large field suppression at $10 \mathrm{~T}$ compared to that observed in $\kappa_{x x}$, excluding a possibility of suppressing the number of spin excitations by a fieldinduced gap. Also, $\ell_{\mathrm{sp}}$ is known to become longer under a magnetic field, because spin fluctuations are suppressed under a magnetic field. Therefore, the field suppression of $\kappa_{x x}^{\mathrm{sp}}$ is caused by a field-suppression effect on $v_{\text {sp }}$. A similar field-suppression effect on $v_{\mathrm{sp}}$ is shown in the 1D spinchain compound [57]. Compared to the 1D spin-chain case, where the elementary excitations are well understood by 
the Bethe ansatz, the spin excitations in a spin liquid state of a 2D kagome are an extremely nontrivial issue. However, from the very similar field-suppression effect on $v_{\text {sp }}$, we suggest the presence of a similar field-suppression effect in $\mathrm{Cd}-\mathrm{K}$ as that in the 1D spin-chain case.

Here, we consider the humplike increase of $\kappa_{x x}$ observed near $T_{N}$. This increase is caused by the increase of $\kappa_{x x}^{\mathrm{ph}}$ by a reduction of spin fluctuations [58] and/or the appearance of a magnon contribution in the ordered state [27]. In the former case, as observed in $\alpha-\mathrm{RuCl}_{3}$ (Ref. [58]), the increase of $\kappa_{x x}$ at $T_{N}$ should be larger under higher fields, because the spin fluctuations are more strongly suppressed under higher fields. However, as shown in Fig. 2, the increase becomes smaller at higher fields. This result is consistent with the field-suppression effect on $\kappa_{x x}^{\mathrm{sp}}$. In addition, the large field suppression of $\kappa_{x x}$ at low temperatures suggests a dominant contribution of $\kappa_{x x}^{\mathrm{sp}}$. Therefore, the increase of $\kappa_{x x}$ below $T_{N}$ is likely attributed to the magnon contribution. The increase of $\kappa_{x x}$ below $T_{N}$ is observed larger in a better crystal with a larger $\kappa_{x x}$. We note that a similar sample dependence of magnon thermal conduction is observed in volborthite [27], which also supports the presence of a magnon contribution below $T_{N}$.

A new field-induced peak is observed in the magnetic field dependence of $\kappa_{x x}$ around $7 \mathrm{~T}$ below $0.3 \mathrm{~K}$ [Fig. 3(c)]. The resonance scattering effect on phonons is excluded to explain the magnetic field dependence, because the resonance scattering effect at $0.3 \mathrm{~K}$ is saturated above approximately $2 \mathrm{~T}$. Also, an increase of $\kappa_{x x}^{\mathrm{ph}}$ by suppressing the AFM phase can be excluded, because $T_{N}$ does not depend on the field up to $10 \mathrm{~T}$ [Fig. 8(a)]. The magnon contribution, which is observed as the humplike increase in $\kappa_{x x}$ below $T_{N}$, is also excluded for the field-induced increase, because the magnon contribution is suppressed by fields (Fig. 2). Therefore, this increase of $\kappa_{x x}$ around $7 \mathrm{~T}$ indicates an appearance of some field-induced spin excitations by closing a spin gap. As shown in Fig. 8(c), the increase of $C_{\mathrm{sp}}$ at $0.5 \mathrm{~K}$ is also observed around $7 \mathrm{~T}$. This increase also supports the appearance of the field-induced spin excitations. Furthermore, in the ordered phase, a finite thermal Hall effect is observed only above $7 \mathrm{~T}$ [Fig. 4(c)], implying that the thermal Hall effect is caused by the field-induced spin excitations observed in the field dependence of $\kappa_{x x}$ and $C$.

One possible origin of this spin gap is an anisotropy of the interactions. In fact, the energy scale caused by $J_{d} / J=$ 0.18 is comparable to $7 \mathrm{~T}$. The temperature dependence of the threshold field of $\Delta T_{y}^{\text {asym }}$ (gray circles in Fig. 9) further supports that the emergence of the field-induced excitations is related to the closing of the spin gap.

\section{B. Thermal Hall conductivity}

\section{Failure of the spin-only model for Cd-K}

We first discuss the temperature dependence of $\kappa_{x y} / T B$ of all three kagome compounds of Cd-K, Ca-K [26], and volborthite [25] in terms of the spin thermal Hall effect calculated by the SBMFT [32,59]. As shown in Fig. 7(b), $\kappa_{x y} / T B$ of these kagome antiferromagnets shows a similar temperature dependence. As reported in Ref. [26], both the temperature dependence and the magnitude of $\kappa_{x y} / T B$ of $\mathrm{Ca}-\mathrm{K}$ and volborthite show good agreement with a simulation based on the SBMFT. In the SBMFT framework, the kagome Heisenberg Hamiltonian with a Zeeman term and a DM interaction is diagonalized by taking a mean-field value of the bond operator of Schwinger bosons. From the energy bands and the Berry curvature calculated by the SBMFT, $\kappa_{x y}^{\mathrm{SBMFT}}$ is calculated by Eq. (1) and is expressed by a dimensionless function $f_{\text {SBMFT }}$ as

$$
\frac{\kappa_{x y}^{\mathrm{SBMFT}}}{T}=\frac{k_{B}^{2}}{\hbar} \frac{D g \mu_{B} B}{J^{2}} f_{\mathrm{SBMFT}}\left(\frac{k_{B} T}{J}\right) .
$$

To compare this SBMFT calculation, the thermal Hall conductivity per one 2D kagome layer is estimated from the experimental data by $\kappa_{x y}^{2 \mathrm{D}}=\kappa_{x y} d$, where $d(d=7.0328 \AA$ for Cd-K [47]) is the distance between the kagome layers. We then compare $\kappa_{x y}^{2 \mathrm{D}}$ with $f_{\mathrm{SBMFT}}$ by normalizing $\kappa_{x y}^{2 \mathrm{D}}$ as

$$
\frac{\kappa_{x y}^{2 \mathrm{D}}}{T}=\frac{k_{B}^{2}}{\hbar} \frac{D g \mu_{B} B}{J^{2}} f_{\mathrm{exp}},
$$

where $J$ and $D$ are the fitting parameters.

By adopting this SBMFT analysis, we fit $\kappa_{x y}^{2 \mathrm{D}}$ of Cd-K by tuning the fitting parameters of $J$ and $D$. Although all the $\kappa_{x y}^{2 \mathrm{D}}$ data of Cd-K well converge to one single curve given by the SBMFT (solid line in Fig. 10) by the fitting parameters listed in Table I, these fittings result in unphysical fitting parameters for Cd-K. First, $J=30 \mathrm{~K}$ used for

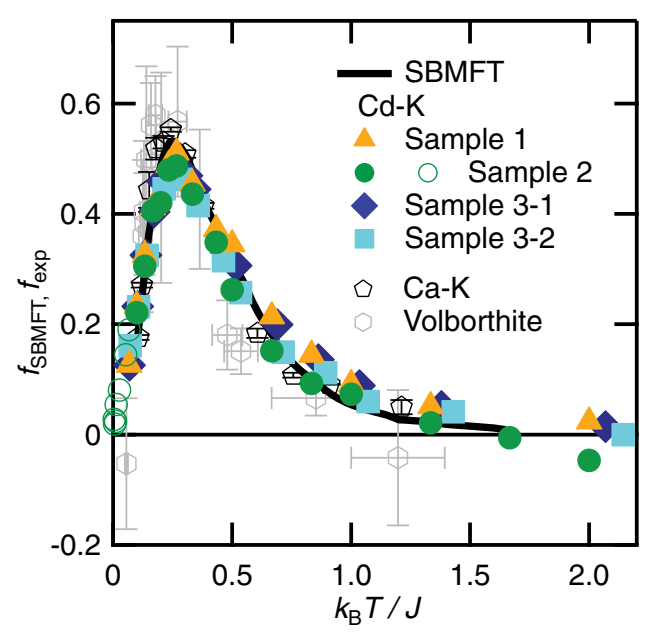

FIG. 10. Normalized thermal Hall conductivity $f_{\text {exp }}$ of kagome lattice antiferromagnets fitted by the parameters listed in Table I. The solid line shows a numerical calculation of $f_{\mathrm{SBMFT}}$ at $D / J=$ 0.1 by the SBMFT [26]. The data of $\mathrm{Ca}-\mathrm{K}$ and those of volborthite are taken from Refs. [26,25], respectively. 
TABLE I. Values of $J$ and $|D / J|$ used to fit $\kappa_{x y}^{2 D}$ to the SBMFT simulation (Fig. 10) for kagome lattice antiferromagnets. The data of $\mathrm{Ca}-\mathrm{K}$ and those of volborthite are taken from Refs. [26,25], respectively.

\begin{tabular}{lccc}
\hline \hline Material & Sample number & $J / k_{B}(\mathrm{~K})$ & $D / J$ \\
\hline Cd-kapellasite & 1 & 30 & 0.28 \\
& 2 & 30 & 0.09 \\
& $3-1$ & 29 & 0.65 \\
Ca-kapellasite [26] & $3-2$ & 28 & 0.6 \\
Volborthite [25] & & 66 & 0.12 \\
\hline \hline
\end{tabular}

the fit of $\mathrm{Cd}-\mathrm{K}$ is considerably smaller than that estimated by the temperature dependence of $\chi(J=45 \mathrm{~K})$ [48]. More importantly, the magnitude of $D$ used to the fit of $\kappa_{x y}$ of $\mathrm{Cd}-\mathrm{K}$ differs by a factor of 7 among the Cd-K samples owing to the very different magnitudes of $\kappa_{x y}$. This large difference of $D$ in Cd-K samples is too large to explain it by the ambiguity in estimating the sample dimensions. In addition, the largest value of $D / J=0.65$ is more than 3 times larger than the value of $D / J \sim 0.19$ estimated from the deviation of the $g$ factor from 2 [48]. This large mismatch is in sharp contrast to the good agreement found in Ca-K [26] in which both $J$ and $D$ determined by the
SBMFT fit of $\kappa_{x y}$ well coincide with the value estimated from the temperature dependence of $\chi$ and that from the deviation of the $g$ factor, respectively. These results indicate that the origin of the thermal Hall effect in $\mathrm{Cd}-\mathrm{K}$ is different from the spin thermal Hall effect observed in Ca-K [26].

\section{Phonon thermal Hall effect in $\mathrm{Cd}-\mathrm{K}$}

As discussed in Sec. IV A, $\kappa_{x x}$ of Cd-K is given by a sum of $\kappa_{x x}^{\mathrm{ph}}$ and $\kappa_{x x}^{\mathrm{sp}}$. Therefore, $\kappa_{x y}$ of Cd-K can also contain a phonon contribution $\kappa_{x y}^{\mathrm{ph}}$ in addition to a spin contribution $\kappa_{x y}^{\mathrm{sp}}$.

Thermal Hall effects of phonons are reported in various compounds [33-37]. In the nonmagnetic insulator $\mathrm{SrTiO}_{3}$, in which only phonons are responsible for the thermal transport, $\kappa_{x y}$ of phonons is found to show a peak at the same temperature of the peak in $\kappa_{x x}$. We thus check this relation for $\mathrm{Cd}-\mathrm{K}$. In $\mathrm{Cd}-\mathrm{K}, \kappa_{x x}$ of $\mathrm{Cd}-\mathrm{K}$ contains a spin contribution $\kappa_{x x}^{\mathrm{sp}}$ which becomes dominant at lower temperatures. On the other hand, as shown in Fig. 3, a magnetic field suppresses a large portion of $\kappa_{x x}^{\mathrm{sp}}$, whereas it slightly increases $\kappa_{x x}^{\mathrm{ph}}$. Therefore, $\kappa_{x x}^{\mathrm{ph}}$ can be estimated by $\kappa_{x x}$ at $15 \mathrm{~T}$.

Figure 11 shows the temperature dependence of $\kappa_{x x} / T$ (left axis) and that of $\kappa_{x y} / T B$ (right axis) at $15 \mathrm{~T}$ of all $\mathrm{Cd}-\mathrm{K}$ (a)

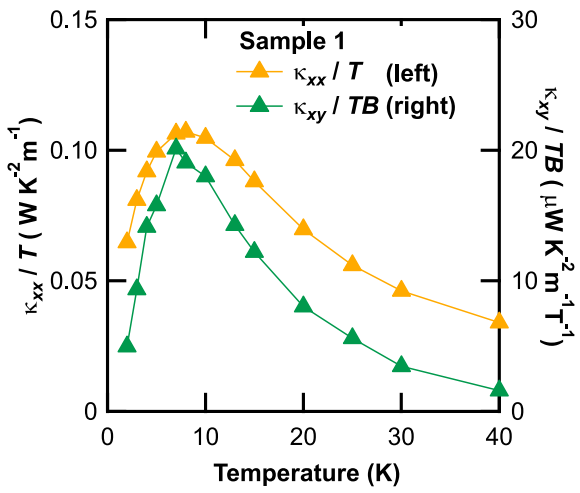

(b)

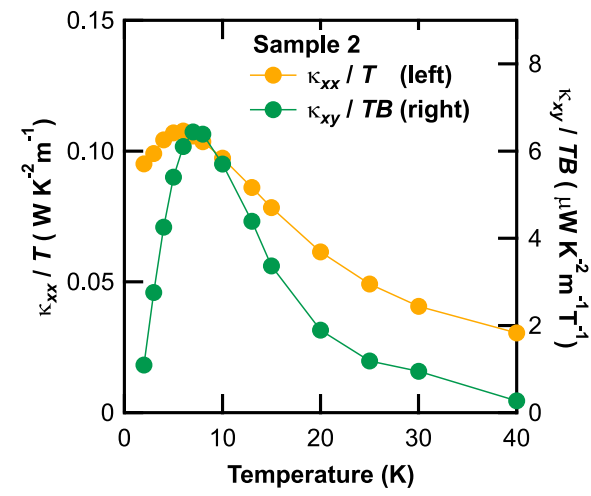

(c)

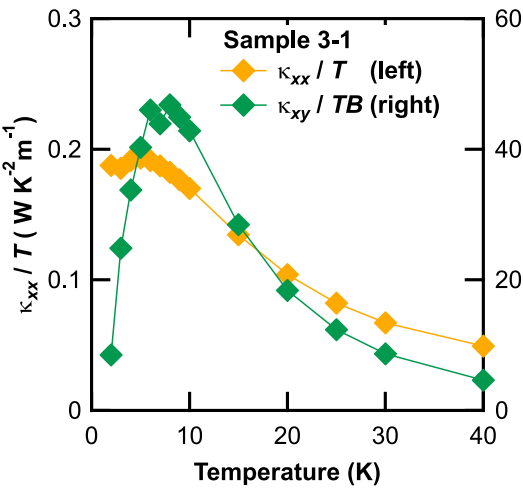

(d)

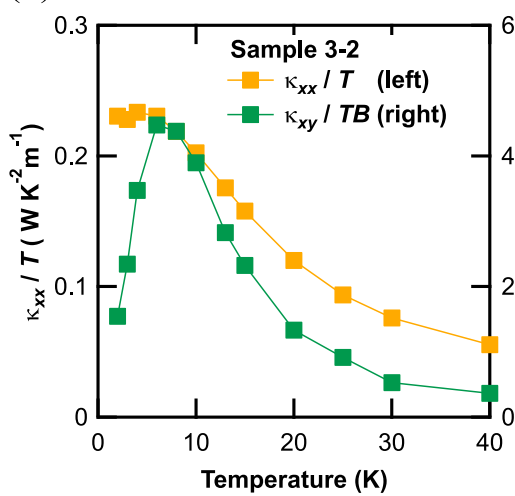

(e)

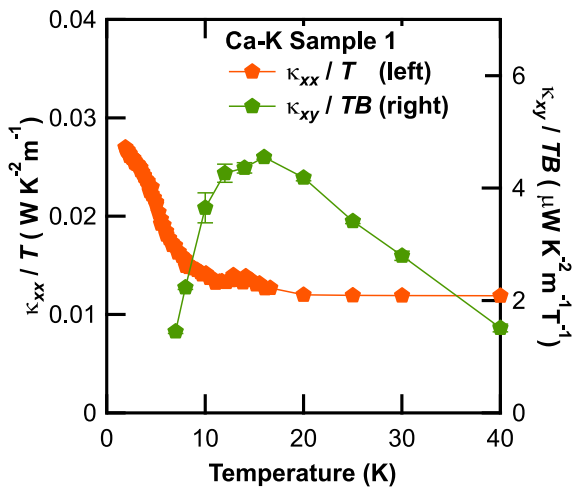

(f)

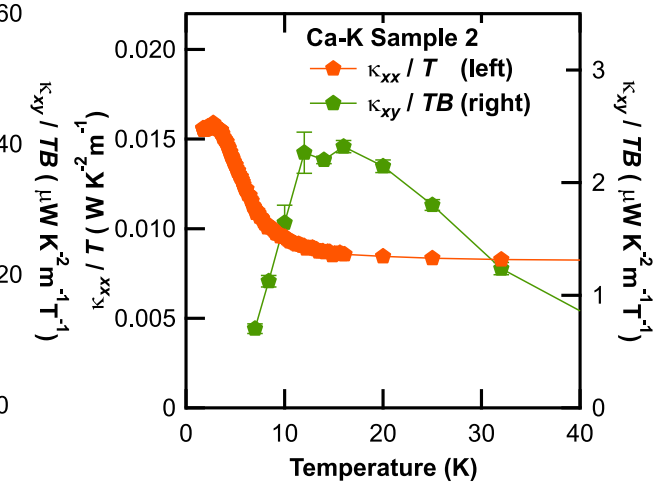

FIG. 11. Temperature dependence of $\kappa_{x x} / T$ (left axis) and that of $\kappa_{x y} / T B$ (right axis) at $15 \mathrm{~T}$ of all Cd-K samples (a)-(d) and Ca-K (e),(f). The data of Ca-K are taken from Ref. [26]. 
samples, together with those of $\mathrm{Ca}-\mathrm{K}$ [26]. As shown in Figs. 11(a)-11(d), $\kappa_{x x} / T$ at $15 \mathrm{~T}$ shows a peak at almost the same temperature of the peak of $\kappa_{x y} / T B$, which resembles the case of the phonon thermal Hall effect observed in $\mathrm{SrTiO}_{3}$ (Ref. [36]). Furthermore, the maximum of $\kappa_{x y} / T B$ almost linearly increases with $\kappa_{x x} / T$, resulting in the estimation of the Hall angle $\left(\kappa_{x y} / \kappa_{x x}\right)$ at the peak temperature as approximately $4 \times 10^{-3}$ at $15 \mathrm{~T}$. This Hall angle is close to that observed in the phonon thermal Hall effects in $\mathrm{SrTiO}_{3}$ [36] and cuprates [37], supporting the phonon origin of $\kappa_{x y}$ in Cd$\mathrm{K}$ at $15 \mathrm{~T}$. Therefore, we conclude that $\kappa_{x y}$ of Cd-K at $15 \mathrm{~T}$ contains a dominant phonon contribution.

We also find that this conclusion is clearly not the case for $\mathrm{Ca}-\mathrm{K}$ [26]. As shown in Figs. 11(e) and 11(f), $\kappa_{x x} / T$ at $15 \mathrm{~T}$ peaks at a much lower temperature than that of $\kappa_{x y} / T B$, which is consistent with the spin origin of $\kappa_{x y}$ in $\mathrm{Ca}-\mathrm{K}$.

\section{Spin thermal Hall effect in Cd-K}

The energy scale of the phonon thermal Hall effect should be given by the Debye temperature, which is estimated as $220 \mathrm{~K}$ for $\mathrm{Cd}-\mathrm{K}$ from the temperature dependence of the specific heat at high temperatures [51]. Since this energy scale is an order of magnitude larger than that of the magnetic field of $15 \mathrm{~T}, \kappa_{x y}^{\mathrm{ph}}$ is expected to have a linear field dependence, which is indeed confirmed in the field dependence of $\kappa_{x y}$ at high temperatures (Fig. 5). On the other hand, the field dependence of $\kappa_{x y}$ becomes nonlinear as lowering temperature. As shown in Fig. 5, the slope of $\kappa_{x y} / B$ below $20 \mathrm{~K}$ becomes larger at lower fields, suggesting an emergence of an additional thermal Hall effect. In this temperature range, $\kappa_{x x}$ also starts to show the field-suppression effect as discussed in Sec. IVA, which is given by the field-suppression effect on $\kappa_{x x}^{\mathrm{sp}}$. Therefore, this nonlinear field dependence of $\kappa_{x y}$ at low temperatures suggests an emergence of a spin contribution $\kappa_{x y}^{\mathrm{sp}}$ which is related to $\kappa_{x x}^{\mathrm{sp}}$.

We estimate this additional spin component $\delta \kappa_{x y}(B)$ at each temperature by

$$
\delta \kappa_{x y}(B)=\kappa_{x y}(B)-\frac{B}{15} \kappa_{x y}(15 \mathrm{~T}) .
$$

Note that $\delta \kappa_{x y}(B)$ gives a lower bound of $\kappa_{x y}^{\mathrm{sp}}$, because the spin contribution in $\kappa_{x y}$ is not fully quenched at $15 \mathrm{~T}$.

We investigate the temperature dependence of $\delta \kappa_{x y} / T B$ at $6 \mathrm{~T}$ (Fig. 12), where the field dependence of $\delta \kappa_{x y}(B)$ shows a peak (see Fig. 5). As shown in Fig. 12, $\delta \kappa_{x y} / T B$ of all the Cd-K samples starts to appear below approximately $25 \mathrm{~K}$, much lower than that of $\kappa_{x y}^{\mathrm{ph}} / T B$ at $15 \mathrm{~T}$ which persists up to approximately $60 \mathrm{~K}$ (Fig. 7). This appearance of $\delta \kappa_{x y} / T B$ coincides with the field-suppression effect on $\kappa_{x x}^{\mathrm{sp}}$ (Fig. 2), indicating that the spin contribution $\kappa_{x y}^{\mathrm{sp}}$ is given by $\delta \kappa_{x y} / T B$.

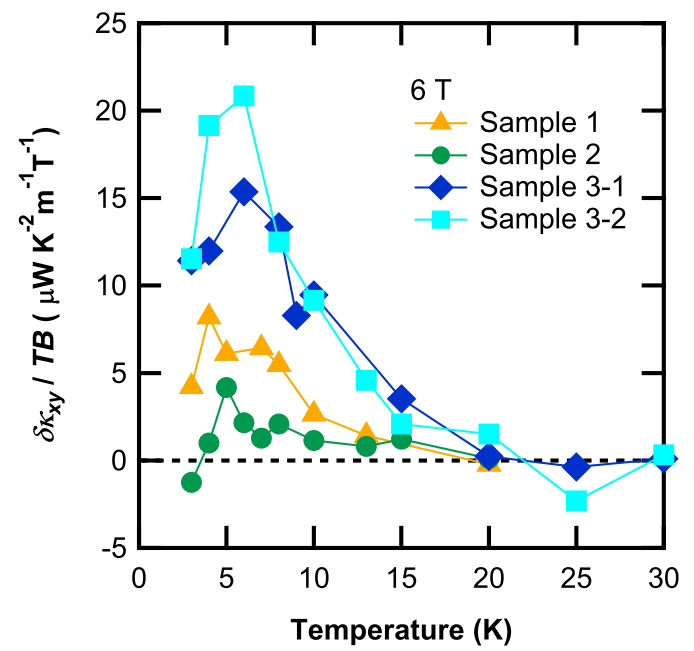

FIG. 12. Temperature dependence of $\delta \kappa_{x y} / T B$ at $6 \mathrm{~T}$ of all Cd-K samples.

Figure 12 shows that the magnitude of $\delta \kappa_{x y} / T B$ depends on the sample, implying that the magnitude of $\kappa_{x y}^{\mathrm{sp}}$ depends on $\kappa_{x x}^{\mathrm{sp}}$. As shown by the field-suppression effect on $\kappa_{x x}^{\mathrm{sp}}$ (Fig. 2), $\kappa_{x x}$ at the peak temperature of $\delta \kappa_{x y} / T B$ contains a significant spin contribution at $0 \mathrm{~T}$. We therefore investigate the dependence of the maximum of $\delta \kappa_{x y} / T B$ on $\kappa_{x x} / T$ at $0 \mathrm{~T}$ at the peak temperature of $\delta \kappa_{x y} / T B$ for each $\mathrm{Cd}-\mathrm{K}$ sample (4-6 K). We also check this relation for Ca-K [26] and volborthite [25]. To compare the results in different samples, we estimate $\kappa_{x y}^{\mathrm{sp}, 2 \mathrm{D}}=\kappa_{x y}^{\mathrm{sp}} \times d$ and assemble all data in Fig. 13 by plotting $\left|\kappa_{x y}^{\mathrm{sp}, 2 \mathrm{D}}\right| / T B$ as a function of $\kappa_{x x} / T$. Most remarkably, as shown in Fig. $13,\left|\kappa_{x y}^{\mathrm{sp}, 2 \mathrm{D}}\right|$ data of three different kagome materials appear to show a smooth function of $\kappa_{x x} / T ;\left|\kappa_{x y}^{\mathrm{sp}, 2 \mathrm{D}}\right|$ stays a constant in the low- $\kappa_{x x}$ region, whereas $\left|\kappa_{x y}^{\mathrm{sp}, 2 \mathrm{D}}\right|$ increases as $\kappa_{x x}$ does. Given that all these compounds share a similar kagome structure (especially for $\mathrm{Ca}-\mathrm{K}$ and $\mathrm{Cd}-\mathrm{K}), \kappa_{x x}\left(\propto C v^{2} \tau\right)$ directly reflects the scattering time $\tau$ determined by disorder scattering. Therefore, Fig. 13 shows $\tau$ dependence of $\left|\kappa_{x y}^{\mathrm{sp}, 2 \mathrm{D}}\right|$.

As discussed in Ref. [26], $\left|\kappa_{x y}^{\mathrm{sp}, 2 \mathrm{D}}\right| / T B$ of $\mathrm{Ca}-\mathrm{K}$ and volborthite can be well explained by the SBMFT calculation $\kappa_{x y}^{\mathrm{SBMFT}} / T B$ of which the magnitude is given only by $D / J$ and does not depend on $\kappa_{x x}$. We denote this as the "intrinsic" contribution (the blue dashed line in Fig. 13). On the other hand, $\left|\kappa_{x y}^{\mathrm{sp}, 2 \mathrm{D}}\right| / T B$ of $\mathrm{Cd}-\mathrm{K}$ samples clearly exceeds the intrinsic contribution and increases as $\kappa_{x x}$ does, which should be denoted as an "extrinsic" contribution (the pink dashed line in Fig. 13).

This $\kappa_{x x}$ dependence of $\left|\kappa_{x y}^{\mathrm{sp}, 2 \mathrm{D}}\right| / T B$ bears similarity to that of the anomalous Hall effect (AHE) in ferromagnetic metals. In the AHE, it is known that the dominant mechanism of AHE depends on the magnitude of the longitudinal conductivity $[60,61]$; the intrinsic mechanism by the Berry curvature of the energy bands is dominant in a 


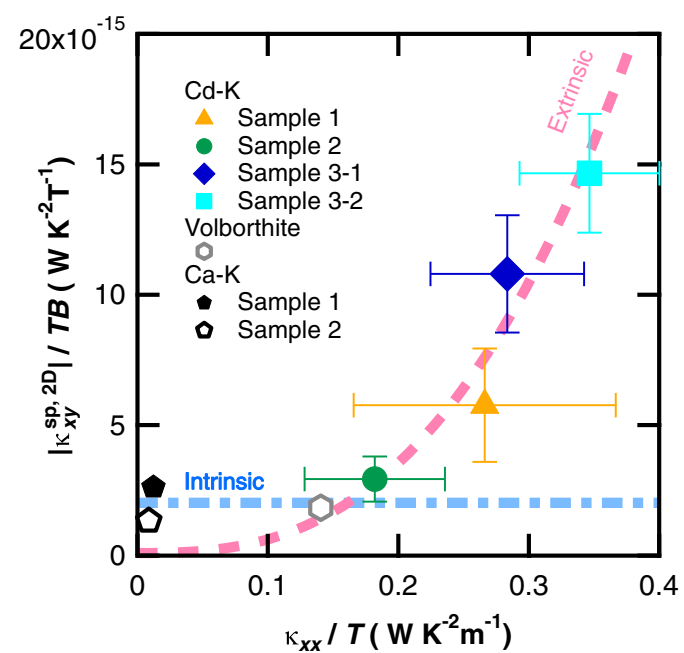

FIG. 13. The spin thermal Hall conductivity per the 2D kagome layer $\left|\kappa_{x y}^{\mathrm{sp}, 2 \mathrm{D}}\right| / T B$ of $\mathrm{Cd}-\mathrm{K}, \mathrm{Ca}-\mathrm{K}$ [26], and volborthite [25] plotted as a function of the longitudinal thermal conductivity $\kappa_{x x} / T$ at $0 \mathrm{~T}$. The value of $\kappa_{x x} / T$ is taken at the peak temperature of $\delta \kappa_{x y} / T B$ for Cd-K (4-6 K; see Fig. 12) and that of $\left|\kappa_{x y}^{2 D}\right| / T B$ for $\mathrm{Ca}-\mathrm{K}$ and volborthite $(16 \mathrm{~K})$. The error bars represent the ambiguity in estimating the absolute value of $\kappa_{x x}$ and $\kappa_{x y}$ owing to the nonrectangular shape of the samples (see Supplemental Material [51] for details). The blue and pink dashed lines are guides to the eye for the intrinsic and extrinsic contributions, respectively (see the main text).

moderate dirty metal, whereas the extrinsic mechanism by skew scatterings is dominant for a superclean metal. This good analogy between the spin thermal Hall effect in the kagome materials and the AHE in ferromagnetic metals indicates the presence of a similar duality of intrinsicextrinsic mechanisms for the spin thermal Hall effect of an insulator.

\section{Spin-phonon coupling and reemergence of $\kappa_{x y}^{\mathrm{ph}}$ in the AFM phase}

In the AFM phase of $\mathrm{Cd}-\mathrm{K}$, no thermal Hall effect is observed below approximately $7 \mathrm{~T}$ [Fig. 4(c)], showing that both $\kappa_{x y}^{\mathrm{ph}}$ and $\kappa_{x y}^{\mathrm{sp}}$ disappear in the AFM phase at low fields. This absence of $\kappa_{x y}^{\mathrm{sp}}$ in the AFM phase is consistent with the theoretical prediction [62]. On the other hand, $\kappa_{x y} / T B$ in the AFM phase reemerges above approximately $7 \mathrm{~T}$ [Fig. 4(c)]. The temperature dependence of $\kappa_{x y} / T B$ at $14 \mathrm{~T}$ below $T_{N}$ well follows that at the spin liquid phase at $15 \mathrm{~T}$ in which the phonon contribution is dominant [see the inset in Fig. 7(b)]. This smooth connection of $\kappa_{x y} / T B$ above and below $T_{N}$ at high fields implies that this highfield $\kappa_{x y}$ below $T_{N}$ is mostly given by phonons. This result is also consistent with that $\kappa_{x y}^{\mathrm{sp}}$ above $T_{N}$ is observed at low fields and the strong field-suppression effect on $\kappa_{x x}^{\mathrm{sp}}$. Therefore, the appearance of $\kappa_{x y}$ in the AFM phase above approximately $7 \mathrm{~T}$ [Figs. 4(c) and 6] shows a reemergence of $\kappa_{x y}^{\mathrm{ph}}$ which disappears at low fields. This absence of $\kappa_{x y}^{\mathrm{ph}}$ in the low-field AFM phase demonstrates that the phonons cannot alone exhibit the thermal Hall effect, putting a strong constraint on the origin of the phonon Hall effect. In other words, $\kappa_{x y}^{\mathrm{ph}}$ needs to be triggered by the field-induced excitations observed in the field dependence of $\kappa_{x x}$ [Fig. 3(c)] and that of $C$ [Fig. 8(b)] through a spin-phonon coupling.

Thermal Hall effects by phonons have been theoretically studied with respect to various aspects [38-46]. The absence of the stand-alone phonon thermal Hall effect in $\mathrm{Cd}-\mathrm{K}$ is inconsistent with an intrinsic mechanism but rather points to extrinsic origins with microscopic couplings between phonons and the field-induced excitations. Such microscopic coupling is also suggested to play an important role in the thermal Hall effect observed in multiferroics [63], where a large thermal Hall effect is observed in the ferrimagnetic phase despite the absence of the conventional magnon Hall effect. Also, it has been pointed out that a magnon-phonon coupling induces a thermal Hall effect even in the system where neither phonons nor magnons alone show a thermal Hall effect [45]. Therefore, the absence and the presence of $\kappa_{x y}^{\mathrm{ph}}$ in the AFM phase of $\mathrm{Cd}-\mathrm{K}$ suggests that the phonon thermal Hall effect in Cd-K has an extrinsic origin requiring a spin-phonon coupling with the field-induced spin excitations. At present, the details of the magnetic structure of Cd-K are not known. Further studies, including NMR or neutron scattering experiments to clarify the magnetic excitations in the AFM phase under the low and the high magnetic fields, will be important to reveal the origins of the thermal Hall effects in Cd-K.

One clearly has to wonder why, despite the dual origin (spins and phonons) of the thermal Hall effects in the Cd-K compounds, the scaling fit derived entirely from the SBMFT works so well as shown in Fig. 10. This conclusion is not unreasonable, however, provided we further assume that $\kappa_{x y}^{\mathrm{sp}}$ is proportional to $\kappa_{x y}^{\mathrm{ph}}$, due to the fact that the two excitations are microscopically coupled.

We note that the field dependence of the thermal Hall effect in the ordered phase of $\mathrm{Cd}-\mathrm{K}$ is in sharp contrast to that observed in $\mathrm{Ca}-\mathrm{K}$. In the ordered phase in $\mathrm{Ca}-\mathrm{K}$, a finite $\kappa_{x y}$ is observed only in a low field and is absent above approximately $6 \mathrm{~T}$ [26], whereas the ordered state in Ca-K is suggested to be the same $q=0$ negative chiral state $[53,64]$. In the two compounds, the magnitude of $\kappa_{x x}$ is quite different (Fig. 2) owing to the different crystal quality. Moreover, according to the theoretical study [62], $\kappa_{x y}^{\mathrm{sp}}$ in the negative chiral state is suggested to depend on the rotation angle of the spin in the kagome plane. Therefore, this different field dependence of $\kappa_{x y}$ could be attributed to the different magnitude of $\kappa_{x x}$ and the spin angle in the AFM state (see Supplemental Material [51] for details). 


\section{SUMMARY}

We have investigated $\kappa_{x x}$ and $\kappa_{x y}$ of three Cd-K samples. From the field-suppression effect on $\kappa_{x x}$, we find that the spin contribution $\kappa_{x x}^{\mathrm{sp}}$ sets in $\kappa_{x x}$ below approximately $25 \mathrm{~K}$, which becomes dominant at lower temperatures. Below $T_{N}$, we find a new peak in the field dependence of $\kappa_{x x}$ at approximately $7 \mathrm{~T}$. Above $7 \mathrm{~T}$, we also find a field-induced increase both in the specific heat and in the thermal Hall effect.

Clear thermal Hall effects have been observed in the spin liquid states of all Cd-K samples. We find that $\kappa_{x y}$ of all Cd$\mathrm{K}$ samples shows a virtually identical temperature dependence with a peak at almost the same temperature. On the other hand, the magnitude of $\kappa_{x y}$ of samples with high $\kappa_{x x}$ substantially exceeds that expected for the intrinsic spin thermal Hall effect determined by $D / J$ in the SBMFT framework. We conclude that a phonon thermal Hall $\kappa_{x y}^{\mathrm{ph}}$ contributes to $\kappa_{x y}$ of all $\mathrm{Cd}-\mathrm{K}$, deduced from the similar temperature dependence of $\kappa_{x y}$ and $\kappa_{x x}$ at $15 \mathrm{~T}$ and the positive correlation between them (Fig. 11).

We further find that the nonlinear field dependence of $\kappa_{x y}$ at low temperatures (Fig. 5) shows the emergence of a spin thermal Hall effect $\kappa_{x y}^{\mathrm{sp}}$ at low fields (Fig. 12). Moreover, the appearance of $\kappa_{x y}^{\mathrm{sp}}$ coincides in its temperature range with the region where the field-suppression effect on $\kappa_{x x}^{\mathrm{sp}}$ is being observed (Fig. 2). Most remarkably, whereas $\kappa_{x y}^{\mathrm{sp}}$ does not depend on $\kappa_{x x}$ and is well explained by the intrinsic thermal Hall effect given by the SBMFT framework in the low- $\kappa_{x x}$ region, $\kappa_{x y}^{\mathrm{sp}}$ exceeds the intrinsic contribution with a positive correlation to $\kappa_{x x}$ for the high- $\kappa_{x x}$ region (Fig. 13). This $\kappa_{x x}$ dependence of $\kappa_{x y}^{\mathrm{sp}}$ points to the presence of both intrinsic and extrinsic mechanisms for the spin thermal Hall effect in the kagome materials, as was the case for the anomalous Hall effects in ferromagnetic metals.

In addition, we find that both $\kappa_{x y}^{\mathrm{ph}}$ and $\kappa_{x y}^{\mathrm{sp}}$ disappear in the AFM phase at low fields. At high fields above $7 \mathrm{~T}$, we find that $\kappa_{x y}^{\mathrm{ph}}$ is induced concomitantly with the field-induced spin excitations observed in the field dependence of $\kappa_{x x}$ and $C$. These results suggest that field-induced spin excitations give rise to a recovery of the phonon thermal Hall effect of Cd-K. We conclude that the phonons alone do not exhibit the thermal Hall effect and require to merge with the fieldinduced spin excitations through a spin-phonon coupling to appear.

\section{ACKNOWLEDGMENTS}

We would like to express our sincere gratitude to the anonymous referees for their valuable comments that helped us to deepen our understanding of the results. H.Y. L. was supported by a Korea University Grant and National Research Foundation of Korea (NRF2020R1I1A3074769). This work was supported by Grants-in-Aid for Scientific Research (KAKENHI) (No. 19H01809, No. 19H01848, and No. 19K21842).
[1] S. Yan, D. A. Huse, and S. R. White, Spin-Liquid Ground State of the $S=1 / 2$ Kagome Heisenberg Antiferromagnet, Science 332, 1173 (2011).

[2] S. Depenbrock, I. P. McCulloch, and U. Schollwöck, Nature of the Spin-Liquid Ground State of the $S=1 / 2$ Heisenberg Model on the Kagome Lattice, Phys. Rev. Lett. 109, 067201 (2012).

[3] H.-C. Jiang, Z. Wang, and L. Balents, Identifying Topological Order by Entanglement Entropy, Nat. Phys. 8, 902 (2012).

[4] Y. Iqbal, F. Becca, S. Sorella, and D. Poilblanc, Gapless Spin-Liquid Phase in the Kagome Spin- $\frac{1}{2}$ Heisenberg Antiferromagnet, Phys. Rev. B 87, 060405(R) (2013).

[5] S. Nishimoto, N. Shibata, and C. Hotta, Controlling Frustrated Liquids and Solids with an Applied Field in a Kagome Heisenberg Antiferromagnet, Nat. Commun. 4, 2287 (2013).

[6] H. J. Liao, Z. Y. Xie, J. Chen, Z. Y. Liu, H. D. Xie, R. Z. Huang, B. Normand, and T. Xiang, Gapless Spin-Liquid Ground State in the $S=1 / 2$ Kagome Antiferromagnet, Phys. Rev. Lett. 118, 137202 (2017).

[7] Y.-C. He, M. P. Zaletel, M. Oshikawa, and F. Pollmann, Signatures of Dirac Cones in a DMRG Study of the Kagome Heisenberg Model, Phys. Rev. X 7, 031020 (2017).

[8] S. Jiang, P. Kim, J. H. Han, and Y. Ran, Competing Spin Liquid Phases in the $S=\frac{1}{2}$ Heisenberg Model on the Kagome Lattice, SciPost Phys. 7, 6 (2019).

[9] S. Sachdev, Kagomé- and Triangular-Lattice Heisenberg Antiferromagnets: Ordering from Quantum Fluctuations and Quantum-Disordered Ground States with Unconfined Bosonic Spinons, Phys. Rev. B 45, 12377 (1992).

[10] F. Wang and A. Vishwanath, Spin-Liquid States on the Triangular and Kagomé Lattices: A Projective-SymmetryGroup Analysis of Schwinger Boson States, Phys. Rev. B 74, 174423 (2006).

[11] L. Messio, S. Bieri, C. Lhuillier, and B. Bernu, Chiral Spin Liquid on a Kagome Antiferromagnet Induced by the Dzyaloshinskii-Moriya Interaction, Phys. Rev. Lett. 118, 267201 (2017).

[12] D. E. Freedman, T. H. Han, A. Prodi, P. Müller, Q.-Z. Huang, Yu.-S. Chen, S. M. Webb, Y.S. Lee, T.M. McQueen, and D. G. Nocera, Site Specific X-Ray Anomalous Dispersion of the Geometrically Frustrated Kagomé Magnet, Herbertsmithite, $\mathrm{ZnCu}_{3}(\mathrm{OH})_{6} \mathrm{Cl}_{2}$, J. Am. Chem. Soc. 132, 16185 (2010).

[13] H. Katsura, N. Nagaosa, and P. A. Lee, Theory of the Thermal Hall Effect in Quantum Magnets, Phys. Rev. Lett. 104, 066403 (2010).

[14] R. Matsumoto, R. Shindou, and S. Murakami, Thermal Hall Effect of Magnons in Magnets with Dipolar Interaction, Phys. Rev. B 89, 054420 (2014).

[15] J. Romhányi, K. Penc, and R. Ganesh, Hall Effect of Triplons in a Dimerized Quantum Magnet, Nat. Commun. 6, 6805 (2015).

[16] J. H. Han, J.-H. Park, and P. A. Lee, Consideration of Thermal Hall Effect in Undoped Cuprates, Phys. Rev. B 99, 205157 (2019).

[17] M. Kawano and C. Hotta, Thermal Hall Effect and Topological Edge States in a Square-Lattice Antiferromagnet, Phys. Rev. B 99, 054422 (2019). 
[18] Y.-f. Yang, G.-M. Zhang, and F.-C. Zhang, Universal Behavior of the Thermal Hall Conductivity, Phys. Rev. Lett. 124, 186602 (2020).

[19] Y. H. Gao and G. Chen, Topological Thermal Hall Effect for Topological Excitations in Spin Liquid: Emergent Lorentz. Force on the Spinons, SciPost Phys. Core 2, 4 (2020).

[20] Y. Teng, Y. Zhang, R. Samajdar, M. S. Scheurer, and S. Sachdev, Unquantized Thermal Hall Effect in Quantum Spin Liquids with Spinon Fermi Surfaces, Phys. Rev. Research 2 , 033283 (2020).

[21] S. Furukawa and T. Momoi, Effects of DzyaloshinskiiMoriya Interactions in Volborthite: Magnetic Orders and Thermal Hall Effect, J. Phys. Soc. Jpn. 89, 034711 (2020).

[22] Y. Onose, T. Ideue, H. Katsura, Y. Shiomi, N. Nagaosa, and Y. Tokura, Observation of the Magnon Hall Effect, Science 329, 297 (2010).

[23] T. Ideue, Y. Onose, H. Katsura, Y. Shiomi, S. Ishiwata, N. Nagaosa, and Y. Tokura, Effect of Lattice Geometry on Magnon Hall Effect in Ferromagnetic Insulators, Phys. Rev. B 85, 134411 (2012).

[24] M. Hirschberger, R. Chisnell, Y. S. Lee, and N. P. Ong, Thermal Hall Effect of Spin Excitations in a Kagome Magnet, Phys. Rev. Lett. 115, 106603 (2015).

[25] D. Watanabe, K. Sugii, M. Shimozawa, Y. Suzuki, T. Yajima, H. Ishikawa, Z. Hiroi, T. Shibauchi, Y. Matsuda, and M. Yamashita, Emergence of Nontrivial Magnetic Excitations in a Spin-Liquid State of Kagomé Volborthite, Proc. Natl. Acad. Sci. U.S.A. 113, 8653 (2016).

[26] H. Doki, M. Akazawa, H.-Y. Lee, J. H. Han, K. Sugii, M. Shimozawa, N. Kawashima, M. Oda, H. Yoshida, and M. Yamashita, Spin Thermal Hall Conductivity of a Kagome Antiferromagnet, Phys. Rev. Lett. 121, 097203 (2018).

[27] M. Yamashita, M. Akazawa, M. Shimozawa, T. Shibauchi, Y. Matsuda, H. Ishikawa, T. Yajima, Z. Hiroi, M. Oda, H. Yoshida, H.-Y. Lee, J. H. Han, and N. Kawashima, ThermalTransport Studies of Kagomé Antiferromagnets, J. Phys. Condens. Matter 32, 074001 (2020).

[28] M. Hirschberger, J. W. Krizan, R. J. Cava, and N. P. Ong, Large Thermal Hall Conductivity of Neutral Spin Excitations in a Frustrated Quantum Magnet, Science 348, 106 (2015).

[29] Y. Kasahara, K. Sugii, T. Ohnishi, M. Shimozawa, M. Yamashita, N. Kurita, H. Tanaka, J. Nasu, Y. Motome, T. Shibauchi, and Y. Matsuda, Unusual Thermal Hall Effect in a Kitaev Spin Liquid Candidate $\alpha-\mathrm{RuCl}_{3}$, Phys. Rev. Lett. 120, 217205 (2018).

[30] Y. Kasahara, T. Ohnishi, Y. Mizukami, O. Tanaka, S. Ma, K. Sugii, N. Kurita, H. Tanaka, J. Nasu, Y. Motome, T. Shibauchi, and Y. Matsuda, Majorana Quantization and Half-Integer Thermal Quantum Hall Effect in a Kitaev Spin Liquid, Nature (London) 559, 227 (2018).

[31] R. Hentrich, M. Roslova, A. Isaeva, T. Doert, W. Brenig, B. Büchner, and C. Hess, Large Thermal Hall Effect in $\alpha-\mathrm{RuCl}_{3}$ : Evidence for Heat Transport by Kitaev-Heisenberg Paramagnons, Phys. Rev. B 99, 085136 (2019).

[32] H. Lee, J. H. Han, and P. A. Lee, Thermal Hall Effect of Spins in a Paramagnet, Phys. Rev. B 91, 125413 (2015).

[33] C. Strohm, G. L. J. A. Rikken, and P. Wyder, Phenomenological Evidence for the Phonon Hall Effect, Phys. Rev. Lett. 95, 155901 (2005).
[34] K. Sugii, M. Shimozawa, D. Watanabe, Y. Suzuki, M. Halim, M. Kimata, Y. Matsumoto, S. Nakatsuji, and M. Yamashita, Thermal Hall Effect in a Phonon-Glass $\mathrm{Ba}_{3} \mathrm{CuSb}_{2} \mathrm{O}_{9}$, Phys. Rev. Lett. 118, 145902 (2017).

[35] Y. Hirokane, Y. Nii, Y. Tomioka, and Y. Onose, Phononic Thermal Hall Effect in Diluted Terbium Oxides, Phys. Rev. B 99, 134419 (2019).

[36] X. Li, B. Fauqué, Z. Zhu, and K. Behnia, Phonon Thermal Hall Effect in Strontium Titanate, Phys. Rev. Lett. 124, 105901 (2020).

[37] G. Grissonnanche, S. Thériault, A. Gourgout, M. E. Boulanger, E. Lefrançois, A. Ataei, F. Laliberté, M. Dion, J. S. Zhou, S. Pyon, T. Takayama, H. Takagi, N. DoironLeyraud, and L. Taillefer, Chiral Phonons in the Pseudogap Phase of Cuprates, Nat. Phys. 16, 1108 (2020).

[38] L. Sheng, D. N. Sheng, and C.S. Ting, Theory of the Phonon Hall Effect in Paramagnetic Dielectrics, Phys. Rev. Lett. 96, 155901 (2006).

[39] Yu. Kagan and L. A. Maksimov, Anomalous Hall Effect for the Phonon Heat Conductivity in Paramagnetic Dielectrics, Phys. Rev. Lett. 100, 145902 (2008).

[40] J.-S. Wang and L. Zhang, Phonon Hall Thermal Conductivity from the Green-Kubo Formula, Phys. Rev. B 80, 012301 (2009).

[41] L. Zhang, J. Ren, J.-S. Wang, and B. Li, Topological Nature of the Phonon Hall Effect, Phys. Rev. Lett. 105, 225901 (2010).

[42] T. Qin, J. Zhou, and J. Shi, Berry Curvature and the Phonon Hall Effect, Phys. Rev. B 86, 104305 (2012).

[43] M. Mori, A. Spencer-Smith, O. P. Sushkov, and S. Maekawa, Origin of the Phonon Hall Effect in Rare-Earth Garnets, Phys. Rev. Lett. 113, 265901 (2014).

[44] T. Saito, K. Misaki, H. Ishizuka, and N. Nagaosa, Berry Phase of Phonons and Thermal Hall Effect in Nonmagnetic Insulators, Phys. Rev. Lett. 123, 255901 (2019).

[45] X. Zhang, Y. Zhang, S. Okamoto, and D. Xiao, Thermal Hall Effect Induced by Magnon-Phonon Interactions, Phys. Rev. Lett. 123, 167202 (2019).

[46] J.-Y. Chen, S. A. Kivelson, and X.-Q. Sun, Enhanced Thermal Hall Effect in Nearly Ferroelectric Insulators, Phys. Rev. Lett. 124, 167601 (2020).

[47] R. Okuma, T. Yajima, D. Nishio-Hamane, T. Okubo, and Z. Hiroi, Weak Ferromagnetic Order Breaking the Threefold Rotational Symmetry of the Underlying Kagome Lattice in $\mathrm{CdCu}_{3}(\mathrm{OH})_{6}\left(\mathrm{NO}_{3}\right)_{2} \cdot \mathrm{H}_{2} \mathrm{O}$, Phys. Rev. B 95, 094427 (2017).

[48] R. Okuma, D. Nakamura, T. Okubo, A. Miyake, A. Matsuo, K. Kindo, M. Tokunaga, N. Kawashima, S. Takeyama, and Z. Hiroi, A Series of Magnon Crystals Appearing under Ultrahigh Magnetic Fields in a Kagomé Antiferromagnet, Nat. Commun. 10, 1229 (2019).

[49] M. P. Shores, E. A. Nytko, B. M. Bartlett, and D. G. Nocera, A Structurally Perfect $S=1 / 2$ Kagomé Antiferromagnet, J. Am. Chem. Soc. 127, 13462 (2005).

[50] R. D. Shannon, Revised Effective Ionic Radii and Systematic Studies of Interatomic Distances in Halides and Chalcogenides, Acta Crystallogr. Sect. A 32, 751 (1976).

[51] See Supplemental Material at http://link.aps.org/ supplemental/10.1103/PhysRevX.10.041059 for (1) errors in estimating the geometrical factors, (2) heater power dependence of the longitudinal and transverse temperature 
differences, (3) temperature stability of the thermal conductivity and the thermal Hall measurements, (4) estimation of the phonon specific heat, and (5) the different field dependence of $\kappa_{x y}$ in the AFM phase of Cd-K and Ca-K.

[52] E. Kermarrec, A. Zorko, F. Bert, R. H. Colman, B. Koteswararao, F. Bouquet, P. Bonville, A. Hillier, A. Amato, J. van Tol, A. Ozarowski, A. S. Wills, and P. Mendels, Spin Dynamics and Disorder Effects in the $S=\frac{1}{2}$ Kagome Heisenberg Spin-Liquid Phase of Kapellasite, Phys. Rev. B 90, 205103 (2014).

[53] Y. Ihara, H. Yoshida, K. Arashima, M. Hirata, and T. Sasaki, Anisotropic Magnetic Excitations from Single-Chirality Antiferromagnetic State in Ca-Kapellasite, Phys. Rev. Research 2, 023269 (2020).

[54] We note that decomposing the thermal conductivity (for both $\kappa_{x x}$ and $\kappa_{x y}$ ) into two independent phonon and spin contributions is an assumption made for convenience of discussion. For a full understanding of the results, one needs to incorporate a merged contribution given by a spinphonon coupling. However, given that the energy scale of a spin-phonon coupling in $\mathrm{Cu}$-based compounds is usually smaller than that of phonons (the Debye temperature) or the spin interaction energy, this two-channel model is often a good approximation.

[55] R. Berman, Thermal Conduction in Solids, Oxford Studies in Physics (Clarendon, Oxford, 1976).

[56] H. Yoshida, N. Noguchi, Y. Matsushita, Y. Ishii, Y. Ihara, M. Oda, H. Okabe, S. Yamashita, Y. Nakazawa, A. Takata, T. Kida, Y. Narumi, and M. Hagiwara, Unusual Magnetic State with Dual Magnetic Excitations in the Single Crystal of $S=$ $1 / 2$ Kagome Lattice Antiferromagnet $\mathrm{CaCu}_{3}(\mathrm{OH})_{6} \mathrm{Cl}_{2}$. 0.6 $\mathrm{H}_{2} \mathrm{O}$, J. Phys. Soc. Jpn. 86, 033704 (2017).

[57] A. V. Sologubenko, K. Berggold, T. Lorenz, A. Rosch, E. Shimshoni, M. D. Phillips, and M. M. Turnbull,
Magnetothermal Transport in the Spin- $\frac{1}{2}$ Chains of Copper Pyrazine Dinitrate, Phys. Rev. Lett. 98, 107201 (2007).

[58] R. Hentrich, A. U. B. Wolter, X. Zotos, W. Brenig, D. Nowak, A. Isaeva, T. Doert, A. Banerjee, P. Lampen-Kelley, D. G. Mandrus, S. E. Nagler, J. Sears, Y.-J. Kim, B. Büchner, and C. Hess, Unusual Phonon Heat Transport in $\alpha-\mathrm{RuCl}_{3}$ : Strong Spin-Phonon Scattering and FieldInduced Spin Gap, Phys. Rev. Lett. 120, 117204 (2018).

[59] J. H. Han and H. Lee, Spin Chirality and Hall-Like Transport Phenomena of Spin Excitations, J. Phys. Soc. Jpn. 86, 011007 (2017).

[60] S. Onoda, N. Sugimoto, and N. Nagaosa, Intrinsic versus Extrinsic Anomalous Hall Effect in Ferromagnets, Phys. Rev. Lett. 97, 126602 (2006).

[61] S. Onoda, N. Sugimoto, and N. Nagaosa, Quantum Transport Theory of Anomalous Electric, Thermoelectric, and Thermal Hall Effects in Ferromagnets, Phys. Rev. B 77, 165103 (2008).

[62] A. Mook, J. Henk, and I. Mertig, Thermal Hall Effect in Noncollinear Coplanar Insulating Antiferromagnets, Phys. Rev. B 99, 014427 (2019).

[63] T. Ideue, T. Kurumaji, S. Ishiwata, and Y. Tokura, Giant Thermal Hall Effect in Multiferroics, Nat. Mater. 16, 797 (2017).

[64] K. Iida, H. K. Yoshida, A. Nakao, H. O. Jeschke, Y. Iqbal, K. Nakajima, S. Ohira-Kawamura, K. Munakata, Y. Inamura, N. Murai, M. Ishikado, R. Kumai, T. Okada, M. Oda, K. Kakurai, and M. Matsuda, $q=0$ Long-Range Magnetic Order in Centennialite $\mathrm{CaCu}_{3}(\mathrm{OD})_{6} \mathrm{Cl}_{2} \cdot 0.6 \mathrm{D}_{2} \mathrm{O}$ : A Spin- $\frac{1}{2}$ Perfect Kagome Antiferromagnet with $J_{1}-J_{2}-J_{d}$, Phys. Rev. B 101, 220408(R) (2020). 